\title{
Mechanical properties of cultured human airway smooth muscle cells from 0.05 to $0.4 \mathrm{~Hz}$
}

\author{
GEOFFREY N. MAKSYM, ${ }^{1}$ BEN FABRY, ${ }^{2}$ JAMES P. BUTLER,${ }^{2}$ DANIEL NAVAJAS, ${ }^{3}$ \\ DANIEL J. TSCHUMPERLIN, ${ }^{2}$ JOHANNE D. LAPORTE, ${ }^{2}$ AND JEFFREY J. FREDBERG ${ }^{2}$ \\ ${ }^{1}$ School of Biomedical Engineering, Dalhousie University, Halifax, Nova Scotia, Canada B3J 3J5; \\ ${ }^{2}$ Physiology Program, Harvard School of Public Health, Boston, Massachusetts 02115; and \\ ${ }^{3}$ Unitat Biofisica i Bioenginyeria, Universitat de Barcelona, 08036 Barcelona, Spain
}

Received 11 May 2000; accepted in final form 21 June 2000

\begin{abstract}
Maksym, Geoffrey N., Ben Fabry, James P. Butler, Daniel Navajas, Daniel J. Tschumperlin, Johanne D. Laporte, and Jeffrey J. Fredberg. Mechanical properties of cultured human airway smooth muscle cells from 0.05 to 0.4 Hz. J Appl Physiol 89: 1619-1632, 2000.-We investigated the rheological properties of living human airway smooth muscle cells in culture and monitored the changes in rheological properties induced by exogenous stimuli. We oscillated small magnetic microbeads bound specifically to integrin receptors and computed the storage modulus $\left(\mathrm{G}^{\prime}\right)$ and loss modulus $\left(\mathrm{G}^{\prime \prime}\right)$ from the applied torque and the resulting rotational motion of the beads as determined from their remanent magnetic field. Under baseline conditions, $G^{\prime}$ increased weakly with frequency, whereas $G^{\prime \prime}$ was independent of the frequency. The cell was predominantly elastic, with the ratio of $G^{\prime \prime}$ to $G^{\prime}$ (defined as $\eta$ ) being $\sim 0.35$ at all frequencies. $\mathrm{G}^{\prime}$ and $\mathrm{G}^{\prime \prime}$ increased together after contractile activation and decreased together after deactivation, whereas $\eta$ remained unaltered in each case. Thus elastic and dissipative stresses were coupled during changes in contractile activation. $\mathrm{G}^{\prime}$ and $\mathrm{G}^{\prime \prime}$ decreased with disruption of the actin fibers by cytochalasin $\mathrm{D}$, but $\eta$ increased. These results imply that the mechanisms for frictional energy loss and elastic energy storage in the living cell are coupled and reside within the cytoskeleton.

cytoskeleton; storage modulus; viscoelasticity; contraction; structural damping; magnetic twisting cytometry
\end{abstract}

PHYSICAL FORCES ACTING ON cells and the resulting cell deformations affect critical features of cell function, including proliferation, differentiation, wound healing, protein and DNA synthesis, apoptosis, cell shape, and cell motility $(3,5,6,22)$. When deformed, a cell stores mechanical energy, and this stored energy permits subsequent recovery of shape. The cell also dissipates

Address for reprint requests and other correspondence: G. N. Maksym, School of Biomedical Engineering, Faculties of Engineering and Medicine, Dalhousie Univ., Halifax, NS, Canada B3H 3J5 (E-mail: gmaksym@is.dal.ca). mechanical energy through mechanical friction. The structural origin for cell elasticity is widely believed to originate in the network of actin fibers within the cytoskeleton (50). However, the origin for energy dissipation is less clear but is often thought to arise from viscous mechanisms associated with shear of the cytoplasmic fluids $(2,26,45,51)$.

A common method to separate elastic from dissipative behavior for any material is to measure responses to oscillatory loads (21). Although the oscillatory mechanics of reconstituted gels of the cytoskeletal filaments have been well studied $(24,32,36)$, there have been relatively few investigations of the oscillatory mechanics of the intact living cell. In studies of the oscillatory mechanics, the cell was probed with the use of a variety of techniques: through the cell surface by atomic force microscopy (46), from the interior by oscillating intracellular granuoles using laser tracking (60), along the cell longitudinal axis using glass manipulators (47), or in a cell pellet using oscillating disk rheometry $(12,23)$. However, no study has yet reported the oscillatory mechanics of the cell by accessing the cytoskeleton through direct attachments to focal adhesions. Focal adhesions are the mechanical connections linking the cytoskeleton of the adherent cell to the extracellular matrix and are the main sites of force transmission between the cytoskeleton and surrounding tissues (30).

We report here a technique for probing the oscillatory mechanics of the cell by direct deformation of the cytoskeleton through focal adhesions. We used ligandcoated magnetic beads $(4.5 \mu \mathrm{m})$, which are bound to the cytoskeleton via integrin receptors, following the method of Wang et al. (55). The beads were magne-

\footnotetext{
The costs of publication of this article were defrayed in part by the payment of page charges. The article must therefore be hereby marked "advertisement" in accordance with 18 U.S.C. Section 1734 solely to indicate this fact.
} 
tized, and an oscillating torque was then applied to the bead by a sinusoidal magnetic field. The resulting oscillating bead rotation was measured by changes in the remanent magnetic field. Using this method, we determined the time courses of both elastic and dissipative mechanical properties of the cell, as well as their frequency dependence.

We found that the human airway smooth muscle (HASM) cell was predominantly elastic, with frictional stresses being about one-third as great as the elastic stresses. Under baseline conditions, the elastic component of cell stiffness, known as the storage modulus $\left(\mathrm{G}^{\prime}\right)$, increased weakly with frequency, whereas the component of the stiffness attributable to friction, the loss modulus $\left(\mathrm{G}^{\prime \prime}\right)$, was independent of the oscillation frequency. Moreover, despite large alterations in $\mathrm{G}^{\prime}$ and $\mathrm{G}^{\prime \prime}$ that we induced by exposure to contractile and relaxing agonists, the amplitude of frictional stresses was a nearly constant fraction of the amplitude of the elastic stresses and nearly independent of frequency. This fraction was altered, however, by disruption of the actin cytoskeleton by cytochalasin $\mathrm{D}$. These data are not consistent with cyclic "peeling" and attachment of the bead at the sites of the adhesion molecules. Moreover, these rheological measurements suggest that the principal frictional stresses that dissipate mechanical energy in the cell do not arise from cytoplasmic fluid flow but rather are coupled to the elastic stresses within the cytoskeleton.

\section{MATERIALS AND METHODS}

\section{Magnetic Oscillatory Cytometry}

The geometry of the system for magnetic oscillatory cytometry is illustrated in Fig. 1. Beads are attached to cells by the methods described below. The beads were magnetized by a brief magnetic pulse $(<0.1 \mathrm{~ms})$ large enough to permanently magnetize the beads $[>0.1$ tesla $(\mathrm{T})]$ oriented at $45^{\circ}$ to the horizontal. We then applied a spatially uniform, time $(t)$ varying magnetic field, $H_{z}(t)$, in the vertical direction, which we will refer to as the twisting field. This field applied a

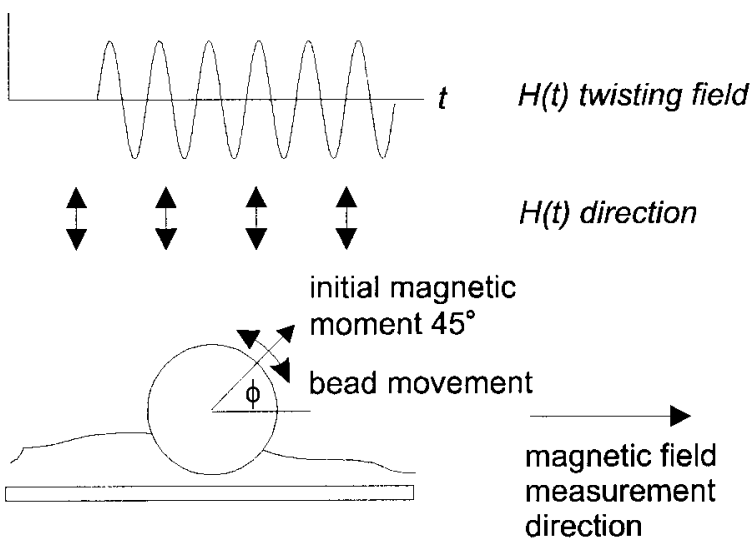

Fig. 1. Schematic of oscillatory magnetic twisting cytometry. The magnetization direction is at $45^{\circ}$ to the vertically applied twisting field. Measuring the horizontal component of the bead's remanent field senses the angular rotation of the beads. $H(t)$, time $(t)$-varying magnetic field; $\phi$, angle of rotation. torque on each bead. The specific torque, $T(t)$, is the torque per unit volume divided by a geometric scaling factor ( 6 for a sphere) and is defined as

$$
T(t)=c H_{z}(t) \cos \phi(t)
$$

where $c$ is the bead constant and $\phi(t)$ is the angle of the magnetic moment vector of the bead relative to the horizontal axis. The bead constant ( $c=0.41 \mathrm{~Pa}$ /gauss in this report) is a property of the magnetic saturation magnetizing moment of the beads and their shape. This constant was determined by a calibration experiment in which the beads were subjected to a constant twist in a medium of known viscosity (56). As defined here, $T(t)$ has units of stress, but this is not to be confused with the actual stress conferred to a cell by a bead, although it may be similar in magnitude.

In oscillatory magnetometry, the twisting field was

$$
H_{z}(t)=H_{a} \sin (2 \pi \mathrm{f} t)
$$

where $H_{a}$ is the amplitude of the applied twist (in gauss) and $\mathrm{f}$ is the oscillation frequency. This sinusoidal twisting field causes the beads to rotate back and forth, and the amount of bead rotation was determined by measuring the horizontal component of the remanent magnetic field produced by the beads. This remanent magnetic field was measured by an in-line, four-probe fluxgate magnetometer (Foerster, Reutlingen, Germany) arranged in a second-order gradiometer configuration (54). Thus, from the filtered magnetic field ( $B$; filtering described below), the angle of rotation $\phi(t)$ was determined by

$$
\phi(t)=\cos ^{-1}\left[B(t) / B_{0}\right]
$$

where $B_{\mathrm{o}}$ is the magnitude of the magnetic field from the beads, determined as follows. Let $B(t)$ be the average $B(t)$ during a 20-s recording before the application of a twisting field when $\phi(t)$ is $45^{\circ}$. Then $B_{0}$ is given by $B_{0}=\sqrt{2} B(t)$. Because the beads are initially magnetized at $45^{\circ}$ rather than in the measurement direction $\left(0^{\circ}\right)$, as in step magnetometry (55), the sensitivity to small changes in angle is greatly increased. For example, a $5^{\circ}$ rotation from $45^{\circ}$ produces a change in magnetic field 24 times greater than that produced by a $5^{\circ}$ rotation from horizontal.

In the absence of a twist field, the measured remanent field is observed to decrease slowly in time. This relaxation of the field is thought to be due to random cellular motions and leads to nonhomogenous bead alignments. An additional contribution to altering the bead alignments might arise from small interbead magnetic forces. The relaxation rate was measured for every sample and was never more than $2 \%$ per minute for HASM cells.

\section{Mechanical Moduli Measured by Magnetic Oscillatory Twisting Cytometry}

Each bead rotates in response to the applied specific torque, but that rotation is impeded by the stresses developed by the cell to which it is attached. The ratio of the applied specific torque and the angular rotation of the bead at the oscillation frequency defines a complex modulus of elasticity (G)

$$
G(f)=T(f) / \phi(f)
$$

where the nonitalicized symbols indicate the Fourier domain. $\mathrm{G}(\mathrm{f})$ is commonly separated into its real and imaginary parts as

$$
\mathrm{G}(\mathrm{f})=\mathrm{G}^{\prime}(\mathrm{f})+i \mathrm{G}^{\prime \prime}(\mathrm{f})
$$


where $\mathrm{G}^{\prime}$ is the storage modulus (corresponding to elastic stresses, proportional to the stored mechanical energy) and $\mathrm{G}^{\prime \prime}$ is the loss modulus (corresponding to frictional stresses, proportional to the dissipated mechanical energy), and $i$ is $\sqrt{-1}$. Equivalently, $\mathrm{G}^{\prime}$ is the component of torque that is in phase with $\phi$, and $\mathrm{G}^{\prime \prime}$ is the component of torque that is $90^{\circ}$ out of phase to $\phi$. The ratio of mechanical energy lost (through dissipation) to energy stored is known as the loss tangent (i.e., the tangent of the phase angle between the stress and strain) or equivalently as the hysteresivity $(\eta)(20)$

$$
\eta(f)=G^{\prime \prime}(f) / G^{\prime}(f)
$$

$\mathrm{G}^{\prime}$ and $\mathrm{G}^{\prime \prime}$, as defined by Eq. 5, represent the shear modulus and the loss modulus, respectively, only if the bead is embedded within an infinite isotropic medium. However, the beads are only partially embedded (Fig. 2, top left) and are linked to the cytoskeleton via focal adhesions at discrete points of contact (Fig. 2, top right and bottom). To relate the magnitude of $\mathrm{G}^{\prime}$ to the shear modulus would require a model, and to propose such a model is beyond the focus of this report. However, the method provides rather precise measurements of the relative changes of the mechanical properties with frequency and with time. It also provides accurate estimates of the ratio of $\mathrm{G}^{\prime \prime}$ to $\mathrm{G}^{\prime}$.

\section{Amplitude Modulation and Demodulation by Spinning}

To shield the cell well from extraneous magnetic fields, the cell well and magnetometer probes were enclosed in four layers of high-permeability $\mu$-metal shielding. To further increase the sensitivity of the system, the cell well was spun at a fixed frequency of $6.53 \mathrm{~Hz}$ so that synchronous detection techniques could be used, as described below. The stability of the spinning frequency was very good, with the spectral
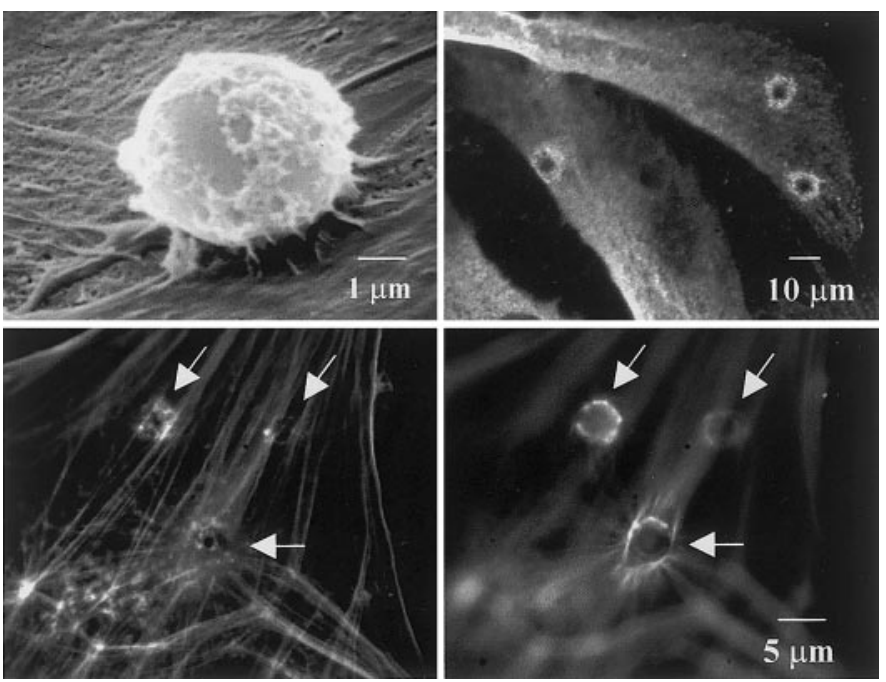

Fig. 2. Top left: electron microscopy of bead bound to an human airway smooth muscle (HASM) cell. Top right: epifluorescence image of HASM cells stained for vinculin with beads 15 min after stimulation with $10^{-5} \mathrm{M}$ histamine and twisting, with a 5.5-Pa amplitude. Bottom: phalloidin staining for actin with beads 7 min after twisting with 5.5-Pa amplitude shown at 2 different focal planes, cutting the basal surface of the cell (left) and focused near the midplane (right). Similar staining for vinculin and actin was apparent after bead adhesion only (no twist or histamine administration), but the staining was less pronounced. Association of actin to the central bead (lower arrows in bottom) is well developed, whereas less developed association is apparent in the beads to the top (top arrows in bottom); 3 beads are shown. spread, expressed as full width at one-half maximum, $<0.00065 \mathrm{~Hz}$

The advantage of spinning the cell well is as follows. The output from the magnetometer, $B_{m}(t)$ (Fig. 3A), contains three components, with only one (induced bead rotation) being of interest. The other two arise from electrical or ambient magnetic noise and a "spill" field component arising from some part of the twisting field detected at the sensor probes due to imperfect alignment; ideally, the perpendicular alignment of the twisting field with the sensor probes avoids the spill artifact. However, due to imperfect geometry and due to the factor of $\sim 10^{6}$ difference in magnitude between the larger applied twisting field vs. the smaller bead remanent field, some spill invariably contaminates the recorded signal. The effect of the spill and a portion of the extraneous noise were removed from the signal by applying a digital, finite impulse response, bandpass filter centered at the spinning frequency (0.6-s width, bandwidth equal to 5 times the oscillation frequency, and Hamming windowed). Figure $3 B$ has an example of the signal $B_{m}(t)$ with the spill field removed $\left[B_{\mathrm{r}}(t)\right]$. Thus frequencies outside a narrow region from the spinning frequency were conveniently rejected. After the bandpass filter was implemented, the magnetic field signal was demodulated using coherent amplitude demodulation on a cycle-by-cycle basis as

$$
=\sqrt{\left[\frac{2}{n} \sum_{\text {cycle }} B_{\mathrm{r}}(t) \sin \left(2 \pi \mathrm{f}_{\mathrm{spin}} t\right)\right]^{2}+\left[\frac{2}{n} \sum_{\text {cycle }} B_{\mathrm{r}}(t) \cos \left(2 \pi \mathrm{f}_{\mathrm{spin}} t\right)\right]^{2}}
$$

where $n$ is the number of data points per cycle and $\mathrm{f}_{\text {spin }}$ is the spinning frequency. The demodulated filtered magnetic field, $B(t)$, is reconstructed by concatenating $B_{\text {cycle }}$ from each spinning cycle and low-pass filtering the reconstructed signal in the Fourier domain, eliminating noise with frequencies greater than four times the oscillation frequency. An example of $B(t)$ and the $T$ - $\phi$ loops calculated using Eqs. 1 and 3 is shown in Fig. $3 C$. In some cases, we applied further filtering to $B(t)$, isolating only the fundamental and the first three harmonics (Fig. $3 D$ ). Note that there is little decrease in $B(t)$ with time (Fig. 3C). With the use of this method for a typical signal strength of $B_{\mathrm{o}}$ from 0.7 to $1.6 \mathrm{nT}$, we can track $\phi(t)$ with a resolution of $10 \mathrm{~s}$, limited by the signal-to-noise ratio, and we can probe the frequency dependence up to $0.5 \mathrm{~Hz}$, limited by the proximity to the spinning frequency, which determines the number of points per oscillation cycle.

\section{Validation With Known Materials}

To assess the ability of the technique to separate elastic behavior from dissipative and to assess the accuracy of the mechanical impedance determined by the oscillatory technique, we tested a fluid with a known viscosity (Petrarch Systems, Bristol, PA). As defined in Eqs. 4 and 5, the magnitude of $G^{\prime \prime}$ is equivalent to the viscosity of a fluid for a completely embedded bead. During oscillatory twisting of beads embedded in the fluid (viscosity $=917 \mathrm{P}$ ), the $T-\phi$ loop was approximately elliptical in shape and had no slope, indicating no elastic behavior. The viscosity was determined as $\mathrm{G}^{\prime \prime} / \omega$ where $\omega=2 \pi \mathrm{f}$. The viscosity was accurately determined for frequencies from 0.05 to $0.4 \mathrm{~Hz}$, for twisting amplitudes between 5 and 60 gauss, and over a range of rotation angles spanning that observed in cells. The error was within $3 \pm 12$ (SD) \% determined from repeated measures. A slight bias was indicated for the highest frequencies $(>0.4 \mathrm{~Hz})$ 
A

B
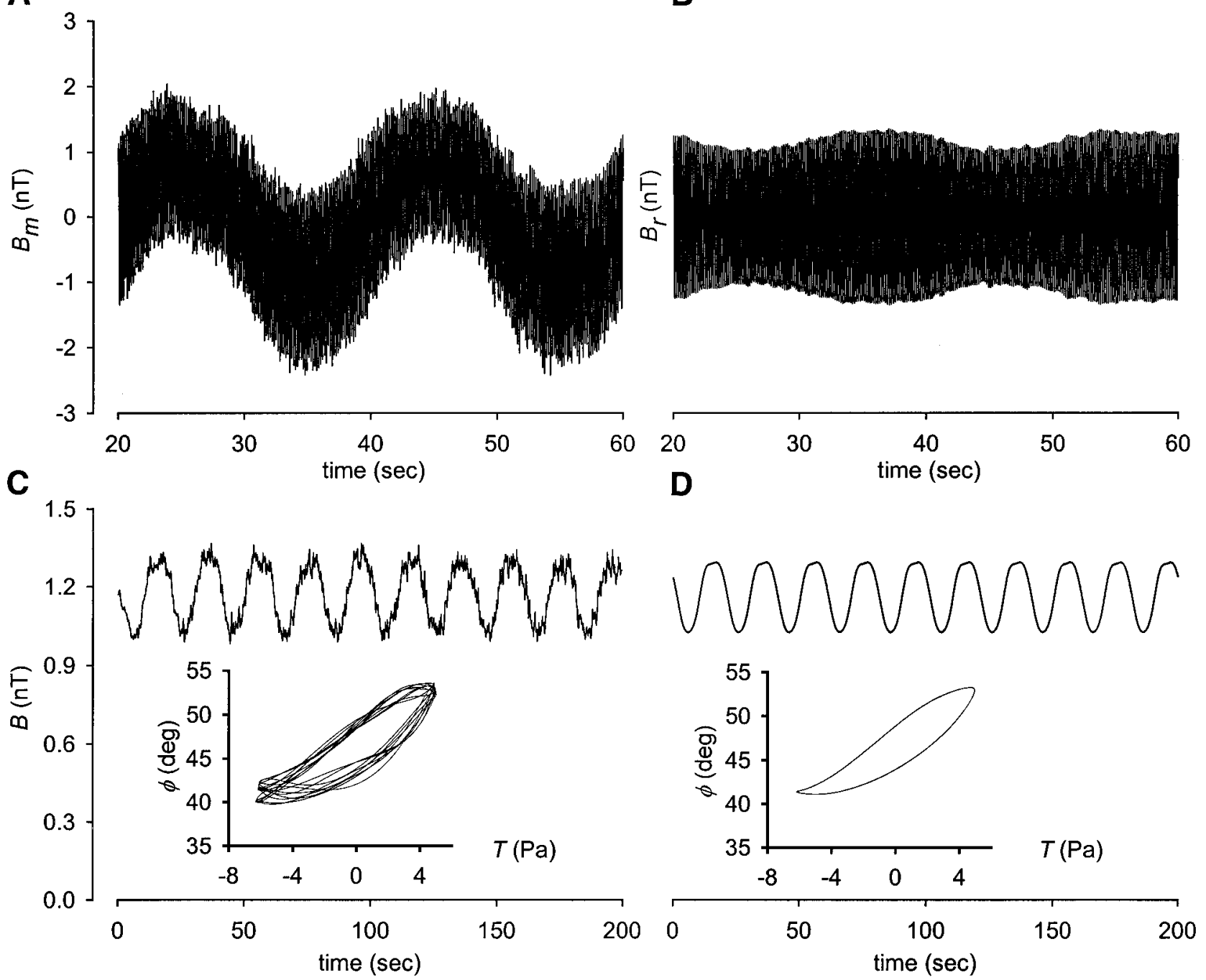

Fig. 3. Demodulation of the magnetic field signal recorded from the beads on HASM cells, which were twisted at $0.05 \mathrm{~Hz}$ and with a spinning frequency of $6.5 \mathrm{~Hz}$. A: raw modulated signal, $B_{m}(t)$, from the magnetometer in nanotesla (nT). $B$ : same signal as in $A$ but band-pass filtered to eliminate the spill artifact from the twisting field $\left(B_{\mathrm{r}}\right)$. This reveals the amplitude modulated signal from the beads. $C$ : Fourier-demodulated signal, $B(t)$; inset shows the calculated torque $(T)-\phi$ loops from the demodulated magnetic field. $D$ : comb-filtered version of the signal, including only the fundamental and the first 3 harmonics; inset shows the corresponding $T-\phi$ loop.

and torque amplitudes ( $>60$ gauss) where the viscosity was overestimated $(\sim 5-10 \%)$, but this bias is not relevant to the results because this torque was outside the range we applied to cells.

Tests for elastic behavior were conducted using beads coated with collagen I (Vitrogen 100, Collagen Biomaterials, Palo Alto, CA), passively attached to polyacrylamide gel (10\% acrylamide with various concentrations of bis-acrylamide; Bio-Rad, Richmond, CA) with the surface activated with 50 $\mathrm{mM}$ sulosuccinimydyl-6-(4' -axido-2'-nitrophenyl-amino)hexanoate (Pierce, Rockford, IL) following the method of Wang and Pelham (58). By varying the concentration of the bisacrylamide, the elasticity of the gel could be varied over several orders of magnitude. The gel was allowed to solidify in individual 96-cell wells, and the beads suspended in PBS were subsequently added to the wells. When subjected to successively increasing step torques, the beads rotated in proportion to the torque; after release of the torque, the rotation fully recovered to the initial angle, verifying elastic behavior in beads bound to the surface of the gel. Beads that were fully embedded could not be bound to the gel and would slip in place, since the interior of the gel could not be activated to bind to collagen. Thus we could not determine the elasticity of the gel with the use of the oscillatory method. However, the sensitivity in estimation of $\mathrm{G}^{\prime}$ could be tested computationally. We superimposed a calculated magnetic signal from beads embedded in an ideal elastic material with a recorded noise signal. The recorded noise was obtained from a sample of beads embedded in epoxy. Thus our model included the effects of spill from $H_{z}(t)$ and magnetic contamination. With the use of $\mathrm{G}^{\prime}$ values of $30-80 \mathrm{~Pa}$ over a range of frequencies from 0.05 to $0.4 \mathrm{~Hz}, \mathrm{G}^{\prime}$ was found to be predicted with an error of $1.7 \pm 2.5 \%$. With the use of these extremes $\left(\mathrm{G}^{\prime}=0\right.$ in the viscous case and $\mathrm{G}^{\prime \prime}=0$ for the elastic case), the limits for the detection of $\eta$ could be determined (Eq. 6). The pure viscosity experimental results set an upper sensitivity limit of $\eta$ equal to $\sim 10$, far above realistic values found in cells and tissues, whereas the elastic simulation predicted a 
minimum sensitivity near $\eta$ equal to $\sim 0.01$, far below that found for the cytoskeleton. Simulations, including both viscosity and elasticity, produced similar accuracy.

\section{Apparent Stiffness by Magnetic Twisting Cytometry}

We compared $\mathrm{G}^{\prime}$ and $\mathrm{G}^{\prime \prime}$ obtained by oscillatory cell twisting to the apparent stiffness $(k)$ obtained by conventional step cell twisting cytometry (56). In the step twist method, $k$ is obtained from the application of a constant twisting field for $1 \mathrm{~min} ; k$ is defined as the ratio of the specific torque at 1 min to the change in angle at $1 \mathrm{~min}$, corrected for the rate of relaxation, and thus is a measure of both elastic deformation and ongoing viscous deformation during the step twist.

\section{Effect of Heterogeneous Bead Rotations}

A factor known to affect the measurement of cell mechanics by magnetic twisting cytometry is that not all beads rotate identically in response to an applied twisting field (14). Heterogeneous bead rotation arises for several reasons, including differences between cells, differences in bead attachment to the cells, local differences within a cell, and magnetic bead-bead interactions. Loosely bound beads tend to rotate more easily and therefore more strongly affect the measured remanent magnetic field. Moreover, the nonlinearity of the arccosine function $(E q .3)$ weights the calculated angular displacement toward beads with greater rotations. All these effects lead to an overestimation of the average rotation during twisting and thus to a large underestimation of the average apparent stiffness (58).

Therefore, we evaluated the degree to which such heterogeneous bead behavior might affect $\mathrm{G}^{\prime}$ and $\mathrm{G}^{\prime \prime}$ and compared the results to the effects on the apparent stiffness as measured by conventional step magnetometry. We simulated the extreme case of a bimodal population of beads, with a stiff population with mechanical parameters $\mathrm{G}_{1}^{\prime}$ and $\mathrm{G}_{1}^{\prime \prime}$, and a compliant or "floppy" population with parameters $\mathrm{G}_{2}^{\prime}$ and $\mathrm{G}_{2}$, with the fraction of floppy to stiff beads determined by a floppy fraction $(\alpha)$. The results for a typical simulation are shown in Fig. 4, left. Because the time constants $\left(\omega \mathrm{G}^{\prime \prime} / \mathrm{G}^{\prime}\right)$ in the model considered here are short compared with the duration of the applied step twist (60 s), the apparent stiff- ness as determined by step magnetometry is a measure of elastic behavior only. From this simulation, it is apparent that step magnetometry can greatly underestimate the elasticity of the stiff population, especially for initial magnetization at $0^{\circ}$ as is conventionally done. This error is reduced if the step twist is done with an initial magnetization of $45^{\circ}$ and is further reduced for oscillatory magnetometry (Fig. 4, left). Thus the mechanics obtained from oscillatory magnetometry much more accurately reflect the average behavior of the heterogeneous population.

This is borne out with measurements of cell mechanics (Fig. 4, right), which compare well with the simulation results. Under control conditions, the apparent stiffness from cells with initial magnetization in the horizontal plane is $50 \%$ of the apparent stiffness with initial magnetization at $45^{\circ}$ and is $20 \%$ of $\mathrm{G}^{\prime}$. The apparent stiffness is less than $\mathrm{G}^{\prime}$, due in large part to the effects of heterogeneous bead rotations but also because apparent stiffness measures both elastic deformation and deformation from ongoing viscous creep during the 60 -s step twist.

Oscillatory magnetometry is also more sensitive to changes in mechanics due to muscle agonists such as endothelin-1 (Fig. 4, right). An increase in $\mathrm{G}^{\prime}$ due to endothelin-1 was $163 \pm 11 \%$ of control, whereas the change in apparent stiffness $\left(0^{\circ}\right.$ magnetization) was lower at $125 \pm 6$ (SE) \%. The reasons for the reduced error and increased sensitivity are largely due to the initial magnetization angle of $45^{\circ}$. Because of the nonlinear cosine function of $E q$. 3, the small rotations from the stiff population contribute very little to $B_{\mathrm{m}}$ when starting from $0^{\circ}$ but contribute much more to $B_{\mathrm{m}}$ when starting from $45^{\circ}$. This is true for both the step twist and oscillatory forcing. However, the results are further improved for oscillatory magnetometry due to smaller oscillation amplitudes (and thus more linear behavior), the fact that the step twist includes ongoing viscous creep, and the fact that $\mathrm{G}^{\prime}$ and $\mathrm{G}^{\prime \prime}$ are calculated only at the driving oscillation frequency. Thus harmonics caused by factors such as loose beads leading to nonlinear distortions of the $T$ - $\phi$ are rejected. In our studies, the harmonics were small, usually $<2 \%$ of the fundamental, but occasionally were as high as $10 \%$.
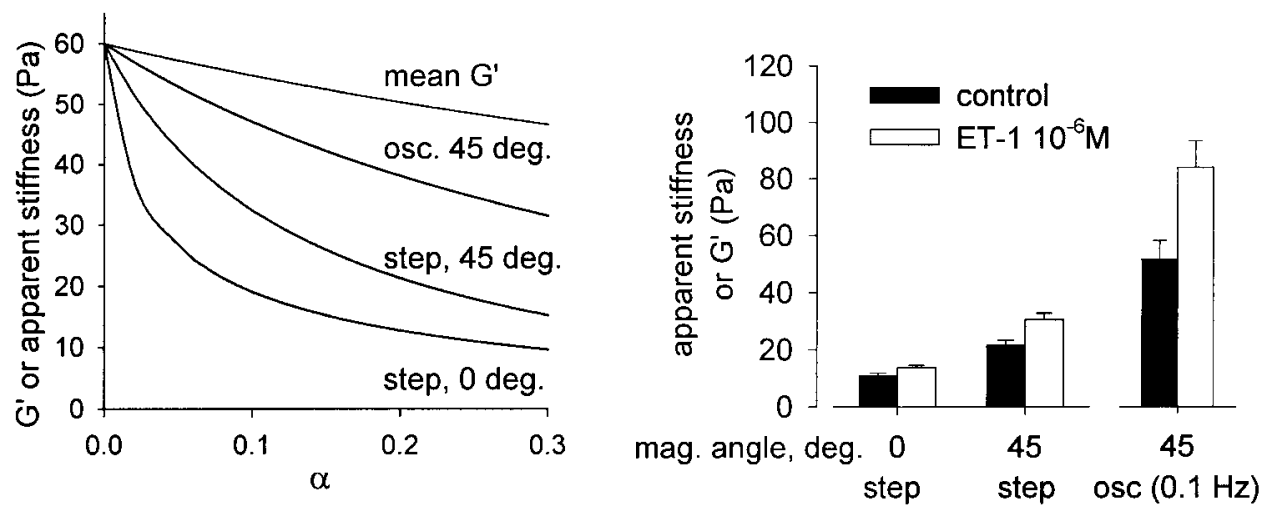

Fig. 4. Left: calculated influence of a loosely bound bead fraction, $\alpha$, to the apparent stiffness determined from step twists $\left(0^{\circ}\right.$ and $45^{\circ}$ initial magnetization) and to the storage modulus $\left(\mathrm{G}^{\prime}\right)$ determined from oscillatory (osc) magnetic twisting cytometry. Mechanical parameters for the population of well-bound beads were $\mathrm{G}^{\prime}=60 \mathrm{~Pa}$ and hysteresivity $(\eta)=0.35$. Parameters for the population of loosely bound beads were $\mathrm{G}^{\prime}=2 \mathrm{~Pa}$ and $\eta=0.35$. The mean $\mathrm{G}^{\prime}$ is calculated from the population as $\mathrm{G}^{\prime}=\mathrm{G}_{\text {stiff }}^{\prime}(1-\alpha)+\alpha \mathrm{G}_{\text {loose }}^{\prime}$. The twisting amplitude was $5.5 \mathrm{~Pa}$; for the oscillatory twist, frequency $=0.1 \mathrm{~Hz}$. Oscillatory simulations and measurements with $45^{\circ}$ initial magnetization angle were the least sensitive to artifacts associated with loosely bound beads. Right: comparison of apparent stiffness from both $0^{\circ}$ and $45^{\circ}$ initial magnetization and $\mathrm{G}^{\prime}$ from oscillatory twisting at $0.1 \mathrm{~Hz}$, amplitude $=5.5 \mathrm{~Pa}$, before and after stimulation with endothelin-1 (ET-1). 
At larger amplitudes of oscillation, these harmonics are larger and lead to greater distortion of the $T-\phi$ loops. Large loops with significant distortions could be used to estimate the quantity of loosely bound beads and also to determine the effect of these beads on the measured mechanical properties. By using least squares fitting of a bimodal population of cell mechanics to the data, we found that the nonlinearity in loop shape can be accounted for by a small number of beads (3.7\%) that are very weakly bound. From a typical $T-\phi$ loop, the two-population-model fit yielded $\mathrm{G}^{\prime}=6 \mathrm{~Pa}$ for the loosely bound bead population and $\mathrm{G}^{\prime}=120.5 \mathrm{~Pa}$ for the stronger bound bead population. To compare, a unimodal population model underestimated the average mechanical properties by only $9 \%$. This underestimate becomes smaller at lower amplitudes of oscillation. This indicates that heterogeneity in bead behavior causes an apparent amplitude dependence for $\mathrm{G}^{\prime}$ and $\mathrm{G}^{\prime \prime}$. Indeed, when we measured the mechanical properties at different magnitudes of oscillation, $\mathrm{G}^{\prime}$ and $\mathrm{G}^{\prime \prime}$ increased by $\sim 40 \%$ for a doubling of the twisting torque amplitude. Because we cannot easily distinguish between the small effects of bead heterogeneity and any amplitude dependence intrinsic to the cytoskeleton, we did not further explore this behavior; throughout this report, we maintained a constant small torque amplitude for individual comparisons $(\leq 5.5 \mathrm{~Pa})$, where the response was nearly linear.

To verify that the mechanics that we determined from the ensemble represented the behavior on a bead-by-bead basis, we also observed the bead motions during oscillation with a microscope. In response to the twisting field, the beads pivoted in a back-and-forth manner, appearing as a translational motion along the line in the direction of the magnetization. We could compare the frequency dependence of mechanical properties at each bead by computing a $G^{\prime}$ and $G^{\prime \prime}$ from the bead translations, rather than from the bead rotations. Within our frequency range, we obtained precisely the same frequency dependence for $\mathrm{G}^{\prime}$ and $\mathrm{G}^{\prime \prime}$ on a bead-by-bead basis as obtained using magnetic detection (Ben Fabry, personal communication). Thus bead heterogeneity does not affect the frequency dependence of the mechanical properties we report, and our data represent the ensemble behavior of the bead movements in response to an applied torque.

Cell culture. Human tracheas were obtained from lung transplant donors in accordance with procedures approved by the University of Pennsylvania Committee on Studies Involving Human Beings. Tracheal smooth muscle cells were harvested from trachea as previously described (37). Briefly, a segment of trachea just proximal to the carina was dissected under sterile conditions, and the trachealis muscle was isolated. Approximately $1 \mathrm{~g}$ of wet tissue was obtained from each donor. The tissue was minced, centrifuged, and resuspended in $10 \mathrm{ml}$ of buffer containing $0.2 \mathrm{mM} \mathrm{CaCl}_{2}, 640$ units collagenase, $10 \mathrm{mg}$ soybean trypsin inhibitor, and 100 units elastase. Tissue was incubated with enzymes for 90 min in a shaking water bath at $37^{\circ} \mathrm{C}$. The cell suspension was then filtered through $127-\mu \mathrm{m}$ Nytex mesh, and the filtrate was washed with an equal volume of cold Ham's F-12 medium supplemented with $10 \%$ fetal bovine serum (FBS). Cells were plated on plastic flasks at $1.0 \times 10^{4} \mathrm{cell} / \mathrm{s} / \mathrm{cm}^{2}$ in Ham's F-12 medium supplemented with $10 \% \mathrm{FBS}, 10^{3} \mathrm{U} / \mathrm{ml}$ penicillin, $1 \mathrm{mg} / \mathrm{ml}$ streptomycin, $2 \mathrm{mg} / \mathrm{ml}$ amphotericin $\mathrm{B}, 12 \mathrm{mM}$ $\mathrm{NaOH}, 1.7 \mu \mathrm{M} \mathrm{CaCl} 2,2 \mathrm{mM}$ L-glutamine, and $25 \mathrm{mM}$ HEPES. Medium was replaced every 3-4 days. Cells were passaged with $0.25 \%$ trypsin and $1 \mathrm{mM}$ EDTA every 10-14 days. Confluent cells were serum deprived and supplemented with $5.7 \mu \mathrm{g} / \mathrm{ml}$ insulin and $5 \mu \mathrm{g} / \mathrm{ml}$ transferrin $24 \mathrm{~h}$ before use. Cells from eight different donors in passages 4-7 were used for this study.
Cell-bead preparation. Cells were washed with $5 \mathrm{ml}$ of PBS and harvested by a brief exposure to $1.5 \mathrm{ml}$ trypsin and EDTA. Bacteriological plastic wells $(6.4 \mathrm{~mm}, 96$-well Removawells, Immunlon II) were prepared for cell plating by coating with collagen I $\left(500 \mathrm{ng} / \mathrm{cm}^{2}\right)$ at least $24 \mathrm{~h}$ before plating. Each well was washed twice with PBS, and the cells were added in 100- $\mu$ l aliquot suspensions at $20 \times 10^{3}$ cells/ well. Between 2.5 and $6 \mathrm{~h}$ later, measurements of cellular mechanics were performed.

The spherical beads (4.5- $\mu \mathrm{m}$ diameter, both generously provided by Dr. W. Moller, Gauting, Germany, and produced at the Harvard School of Public Health) were first coated with a synthetic RGD (Arg-Gly-Asp) containing peptide (Peptite 2000, Integra Life Sciences, San Diego, CA) coated at a density of $50 \mu \mathrm{g}$ of peptide/mg beads in $1 \mathrm{ml}$ of carbonate buffer ( $\mathrm{pH}$ 9.4). The beads were suspended in $1 \%$ serum medium, and 50,000 beads were added to an individual cell well. After a 15 - to 20 -min incubation at $37^{\circ} \mathrm{C}$ to allow binding of the beads to the cells, the well was washed twice with serum-free medium to remove any unbound beads. After adhesion, the cell well was then placed in the magnetic twisting cytometer and maintained at $37^{\circ} \mathrm{C}$ for determination of mechanical properties.

Reagents. Tissue culture reagents and drugs used in this study were obtained from Sigma Chemical (St. Louis, MO), with the exception of amphotericin B and Trypsin-EDTA solution, which were purchased from GIBCO (Grand Island, NY), and endothelin-1, which was obtained from Calbiochem (San Diego, CA). Dibutyryl cAMP (DBcAMP) (a cell-permeant analog to cAMP) was dissolved at $10^{-1} \mathrm{M}$ in distilled water, frozen in aliquots, and thawed on the day of use. Endothelin-1 was dissolved in 5\% acetic acid and distilled water at $10^{-4} \mathrm{M}$, frozen in aliquots, and thawed on the day of use. Histamine was dissolved in distilled water at $10^{-1} \mathrm{M}$, frozen in aliquots, thawed on the day of use, and diluted in medium. Isoproterenol $\left(10^{-1} \mathrm{M}\right.$ in distilled water) was made fresh each day. Because isoproterenol is rapidly oxidized, dilutions of isoproterenol in medium were made immediately before addition to the cells.

\section{Experimental Protocol}

We quantified the mechanical properties of the cells at three or four different oscillation frequencies, before and after administration of pharmacological agonists. In some cases, we also measured the response to the agonists during oscillation. We also measured conventional step twists before and after the agonists, with magnetization angles at both $0^{\circ}$ and $45^{\circ}$

After a measurement of relaxation, the apparent stiffness was measured by step twists from both $0^{\circ}$ magnetization with a 14.1-gauss twisting field and from $45^{\circ}$ magnetization with a 20 -gauss twisting field. The twisting fields were chosen so that the initial specific torques were the same at $\sim 5.5$ $\mathrm{Pa}(E q .1)$. The equivalent force-couple defined by the radius of the bead for this specific torque is $12 \mu \mathrm{dyn}(120 \mathrm{pN})$. This yielded rotational amplitudes of about $5^{\circ}\left(10^{\circ}\right.$ peak to peak) under baseline conditions. Three oscillatory twists were applied in random order of $0.05,0.1$, and $0.2 \mathrm{~Hz}$ with a 20 -gauss twisting field. A pharmacological agonist at a maximally effective dose was then added, which was either a relaxing agonist $\left(10^{-3} \mathrm{M}\right.$ DBcAMP or $10^{-6} \mathrm{M}$ isoproterenol) or a contractile agonist $\left(10^{-5} \mathrm{M}\right.$ endothelin-1 or $10^{-5} \mathrm{M}$ histamine). After administration of the agonist, and a pause of $300 \mathrm{~s}$, the oscillatory twists were repeated in random order, followed by step twists at $45^{\circ}$ magnetization angle and $0^{\circ}$ magnetization angle, with the same twisting amplitude as 
used before the administration of the agonist. The protocol for each sample took $\sim 0.5 \mathrm{~h}$. Oscillatory measurements were made immediately before the addition of cytochalasin $\mathrm{D}$ and $16 \pm 1.8 \mathrm{~min}$ after. Throughout this study, changes in mechanical parameters from control values were determined by paired $t$-test $(P<0.05)$.

To determine mechanics from cell receptors not linked to the cytoskeleton through focal adhesions, we also used beads coated with the acetylated low-density lipoprotein (acLDL), which binds to low-density lipoprotein scavenger receptors in the cell membrane, at a saturation concentration of $50 \mu \mathrm{g} / \mathrm{mg}$ beads (41). The twisting amplitude was reduced to $1.9 \mathrm{~Pa}$, since the rotational amplitudes were excessively large at higher torques. In a further protocol, the time course of change of mechanics with histamine was determined by delivering the agonist during an oscillatory twist measurement. This was done by a micropipette tip attached to a long catheter, which was connected to a 1-ml syringe fitted to the sample holder.

\section{Immunofluorescent Imaging}

Some cells were plated on 12-mm-diameter coverslips for fixation and antibody staining. They were prepared as for the measurements described above and placed in a twisting coil, which reproduced the magnetic twisting conditions in the magnetic twisting cytometer system. After a period of no twisting field (control) or twisting torque amplitude $=5.5 \mathrm{~Pa}$ for 15 min, coverslips were washed twice with PBS and fixed in $2 \%$ paraformaldehyde for $15 \mathrm{~min}$. After two more washes in PBS, they were permeabilized with 0.3\% Triton X-100 (5 min) and again washed twice in PBS. Coverslips were then stained with a 1:40 dilution of alexa 488 phalloidin (Molecular Probes, Eugene, OR) or with a 1:100 dilution of antibody to vinculin (anti-human, mouse IgG1, Sigma Chemical). Both dilutions were in PBS with $0.1 \%$ BSA and $0.05 \%$ Tween 20. Coverslips stained with phalloidin were mounted immediately on a second coverslip (permitting imaging of either basal or apical surface) with ProLong antifade mounting medium (Molecular Probes). Coverslips incubated with antivinculin primary were further incubated with a biotinylated secondary antibody (horse anti-mouse, Sigma Chemical) at a 1:200 dilution and then with streptavidin-conjugated Texas red (1:750 dilution). Coverslips were then mounted identi- cally to those stained with phalloidin. Imaging was done on an Olympus inverted epifluorescence microscope with $\times 10$, $\times 20$, and $\times 100$ (oil immersion) magnifications.

\section{RESULTS}

\section{Tracking the Time Course of Cytoskeletal Mechanics During Histamine Challenge}

The administration of histamine $\left(10^{-5} \mathrm{M}\right)$ caused the amplitude of the oscillating bead rotation to be markedly reduced with a narrowing of the $T-\phi$ loop and a decrease of its slope, indicative of cell stiffening and a decrease in mechanical energy dissipation (Fig. 5). Both $G^{\prime}$ and $G^{\prime \prime}$ increased to maximal values within $40 \mathrm{~s}$, achieving about five times their baseline values, and then decreased gradually and within $100 \mathrm{~s}$ stabilized at steady-state levels (Fig. 6). Hysteresivity became more variable after histamine administration but remained largely unchanged after $100 \mathrm{~s}$.

\section{Frequency Dependence of Cytoskeletal Mechanics}

Under baseline conditions, $\mathrm{G}^{\prime}$ increased with frequency according to a weak power law, $\mathrm{f}^{0.20}$, whereas $\mathrm{G}^{\prime \prime}$ was independent of frequency (Fig. 7, top). The apparent viscosity $\left(\mathrm{G}^{\prime \prime} / \omega\right)$ varied exactly inversely with frequency. Hysteresivity decreased slightly with frequency (Fig. 7, bottom).

With challenge by contractile agonists (endothelin-1 and histamine), $G^{\prime}$ and $G^{\prime \prime}$ were elevated in the steady state (Fig. 8). After relaxant agonists (isoproterenol or DBcAMP) were administered, both $\mathrm{G}^{\prime}$ and $\mathrm{G}^{\prime \prime}$ were decreased (Fig. 9). At all frequencies and for all agonists, $\eta$ was unchanged (Fig. 8 and 9 , right). Thus the energy dissipation due to frictional stresses remained a constant fraction of the energy stored due to elastic stresses, both during smooth muscle activation and during deactivation. Time controls showed a slight increase in $\mathrm{G}^{\prime}$ and $\mathrm{G}^{\prime \prime}$, but the increase was not significant.

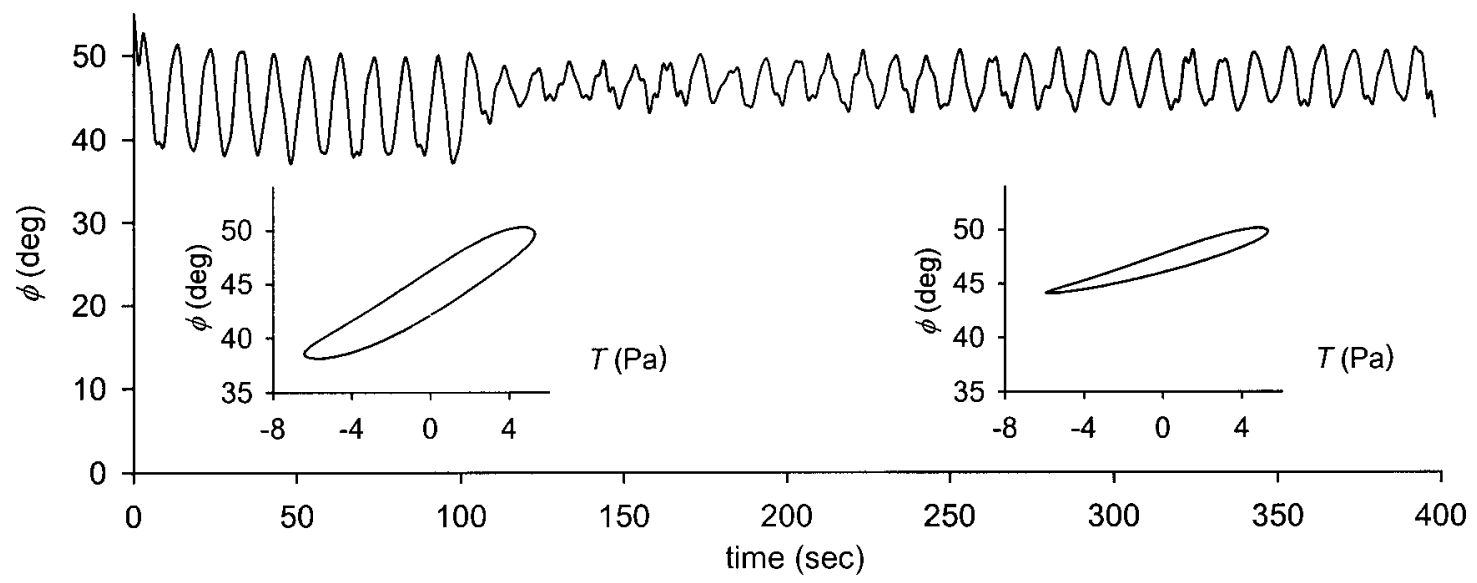

Fig. 5. Example of the angular rotation computed from the measured magnetic field from a single well of HASM cells with beads attached during twisting with a $0.1 \mathrm{~Hz}, 20$-gauss applied twisting field. Histamine $\left(10^{-5} \mathrm{M}\right)$ was added at $100 \mathrm{~s}$. Insets show the $T-\phi$ loops from comb filtering the first 10 cycles and the last 20 cycles (twisting frequency and first 3 harmonics), clearly showing decrease in angular amplitude, decrease in slope, and narrowing of the loop. 

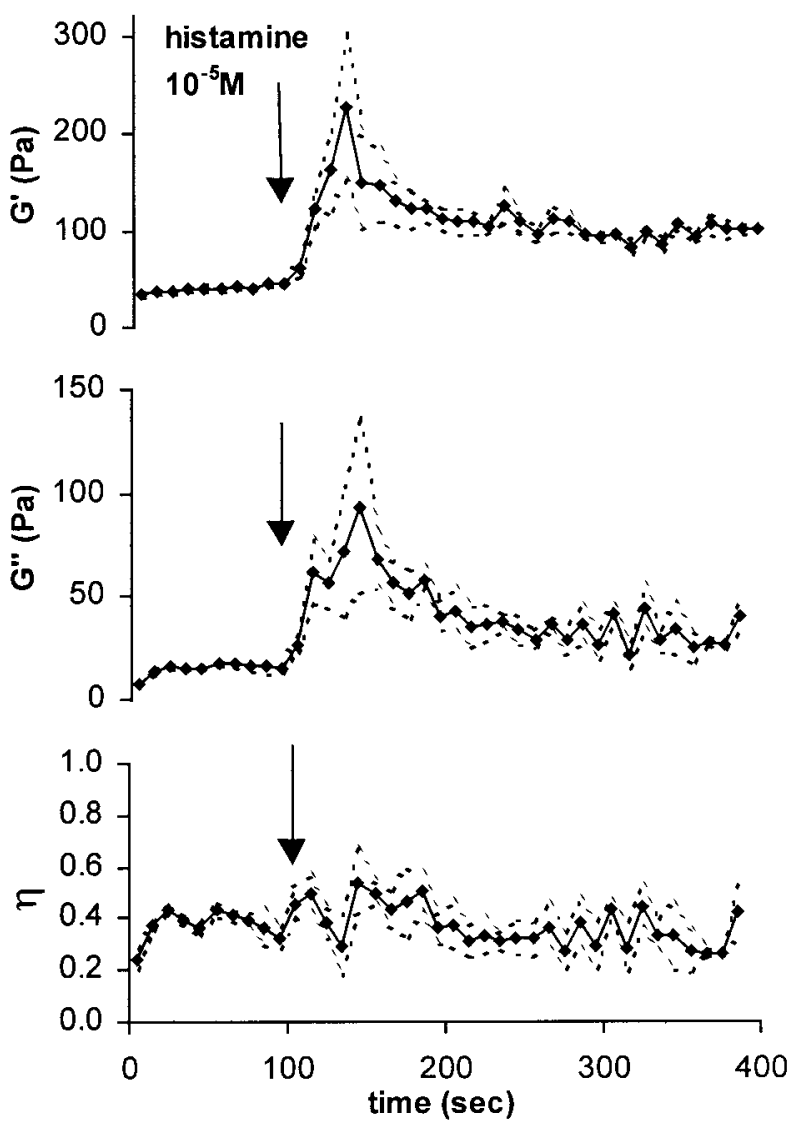

Fig. 6. Time course of the mechanical properties of HASM cells during the administration of histamine $\left(10^{-5} \mathrm{M}\right)$ at $100 \mathrm{~s}$ (dashed lines indicate $\mathrm{SE}, n=5)$. $\mathrm{G}^{\prime}(t o p)$ and loss modulus $\left(\mathrm{G}^{\prime \prime} ;\right.$ middle $)$ are transiently increased before reaching elevated plateau levels, whereas $\eta$ (bottom) becomes more variable but remains relatively unchanged.

Disruption of the cytoskeleton by cytochalasin $\mathrm{D}$ caused a greater decrease in $\mathrm{G}^{\prime}$ than in $\mathrm{G}^{\prime \prime}$ (Fig. 10), unlike the concordant changes in both $G^{\prime}$ and $G^{\prime \prime}$ found during muscle activation or deactivation (Figs. 8 and 9). Hysteresivity was thus increased (Fig. 10, bottom).

The magnitudes of $\mathrm{G}^{\prime}$ and $\mathrm{G}^{\prime \prime}$ measured with beads coated with acLDL were markedly smaller compared with beads bound via the integrin receptors, although the frequency dependence was similar (Fig. 11). Hysteresivity was lower with beads bound via acLDL than with beads bound to integrins via RGD.

\section{DISCUSSION}

The principal findings of this report are as follows. First, under baseline conditions, $\mathrm{G}^{\prime}$ increased weakly with oscillation frequency, whereas $G^{\prime \prime}$ was independent of oscillation frequency, and $\eta$ was close to 0.35 and decreased slightly with frequency. Second, $G^{\prime}$ and $\mathrm{G}^{\prime \prime}$ increased sharply during contractile activation and decreased during deactivation, but $\eta$ remained unchanged. Finally, disruption of actin filaments by cytochalasin $D$ caused a greater decrease in $G^{\prime \prime}$ than in $\mathrm{G}^{\prime}$, leading to an increase in $\eta$. The insensitivity of frictional losses to the frequency of mechanical oscilla- tion and the near constancy of the balance between energy dissipation and energy storage despite changes in muscle activation are novel findings and are not explained by current models of cytoskeleton rheology. In the sections that follow, we discuss the methodology and the principal characteristics of the phenomenology with comparison to other data reported in the literature. We conclude with comments on the implications of these findings for mechanism.

We considered the possibility that the observed behavior might be attributable to cyclic "peeling" and attachment of the bead at the sites of the integrin receptor-RGD ligand bonds. However, there are a number of arguments against this idea. The strong attachment of the beads to the cytoskeleton by RGD-integrin binding is well established $(39,40,55)$, and the development of focal adhesions and complex interconnections to the actin fiber matrix was readily observed at the bead locations (Fig. 2). In addition, both $\mathrm{G}^{\prime}$ and $\mathrm{G}^{\prime \prime}$ increased with stimulation of the contractile apparatus and conversely decreased with relaxation of the contractile machinery. Furthermore, when the actin cytoskeleton was disrupted by cytochalasin $\mathrm{D}, \mathrm{G}^{\prime}$ was greatly decreased, perhaps by the direct destruction of the actin filament network but possibly through disconnection of the cytoskeleton from the integrin molecules attached to the beads. Finally, when we measured the mechanical properties of beads not linked to the cytoskeleton but with beads bound to receptors for low-density lipoprotein that do not form focal adhesions (55), the measured values of $\mathrm{G}^{\prime}$ and $\mathrm{G}^{\prime \prime}$ were
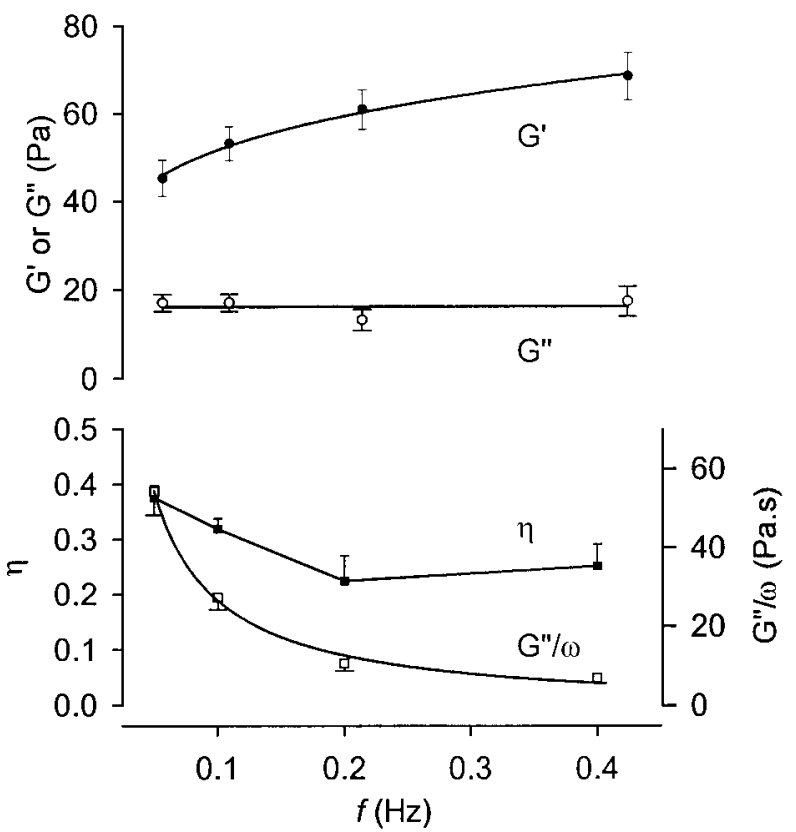

Fig. 7. Frequency (f) dependence of the mechanics of cultured HASM cells under baseline conditions with least squared fit curves. Top: $\mathrm{G}^{\prime}=827 \mathrm{f}^{0.20}, r^{2}=0.995$, and $\mathrm{G}^{\prime \prime}=4 \mathrm{f}+161, r^{2}=0.00095$. The low correlation coefficient is due to the very little variation in $\mathrm{G}^{\prime \prime}$ with frequency. Bottom: apparent viscosity, $\mathrm{G}^{\prime \prime} / \omega=30 / \mathrm{f}-13, r^{2}=0.995$. Data are calculated with specific torque amplitude of $4.2 \mathrm{~Pa}$; error bars indicate SE $(n=14)$. 

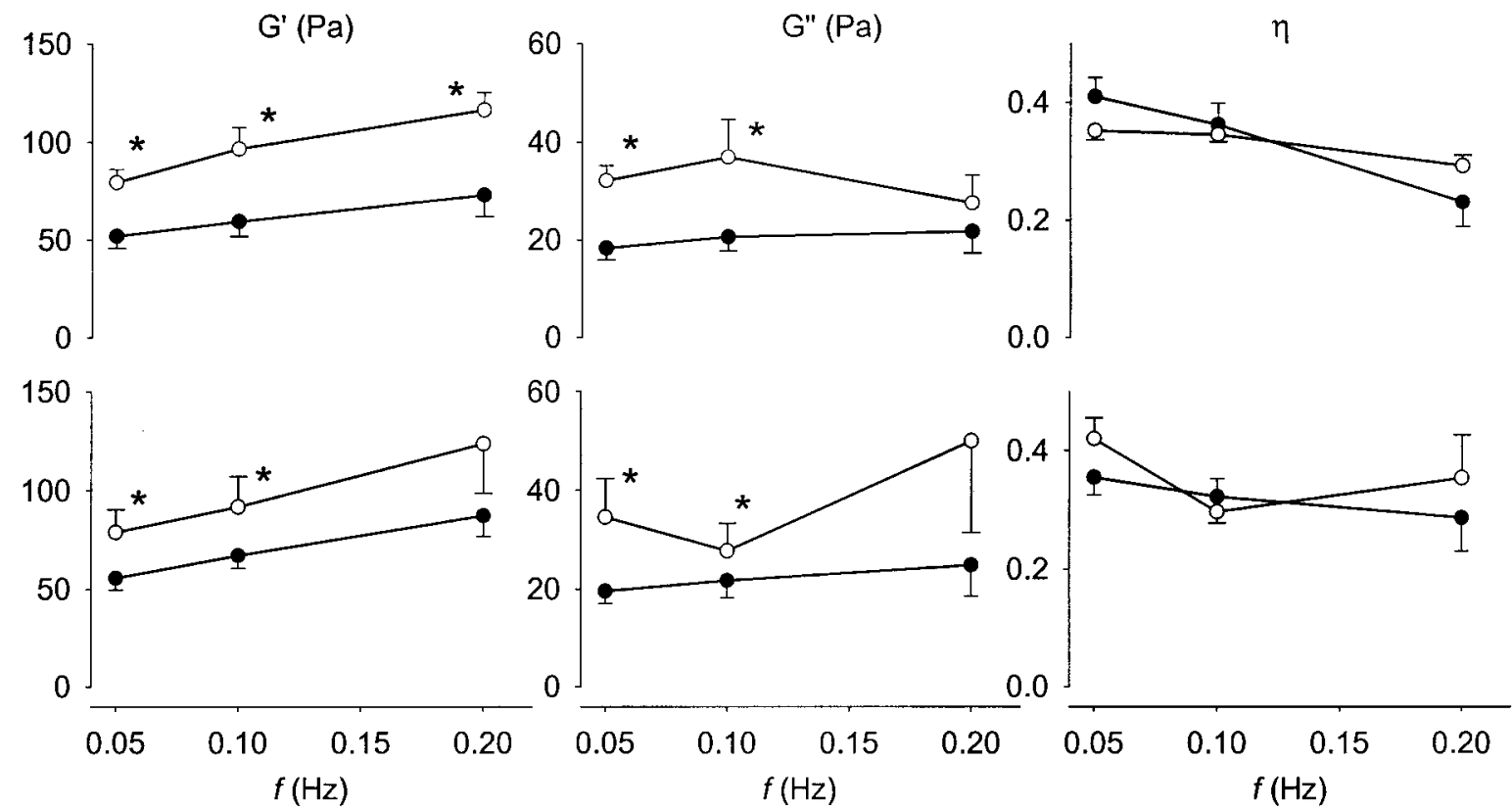

Fig. 8. Control $(\bullet)$ and plateau $(\circ)$ responses from contractile agonists. Top: ET-1. Bottom: histamine. In each case, $\mathrm{G}^{\prime}\left(\right.$ left) and $\mathrm{G}^{\prime \prime}$ (middle) are significantly decreased from control values in nearly all cases $(* P<0.05)$, whereas $\eta$ (right) remains unchanged. Error bars indicate SE; $n=8$ (ET-1) and 5 (histamine).

fivefold smaller than the values found with RGDcoated beads. Together, these observations support the conclusion that the moduli we obtained reflect the mechanics of the cytoskeleton, which we will discuss below in Contractile Responses, and are modulated by activity of the contractile machinery.

\section{Comparison With Previous Findings}

Measurement of cellular mechanics using magnetic particles was used as early as 1950, when Crick and
Hughes $(8,9)$ measured the viscosity and elasticity inside cells by observing how individual internalized ferromagnetic particles rotated in reaction to an applied magnetic field. Valberg (53) extended this technique to populations of particles internalized in populations of cells by measuring the changes in the remanent magnetic field of the magnetic particles as they rotated. More recently, Wang et al. (55) adapted this technique still further by using beads bound to the cytoskeleton via integrin receptors. This method, now
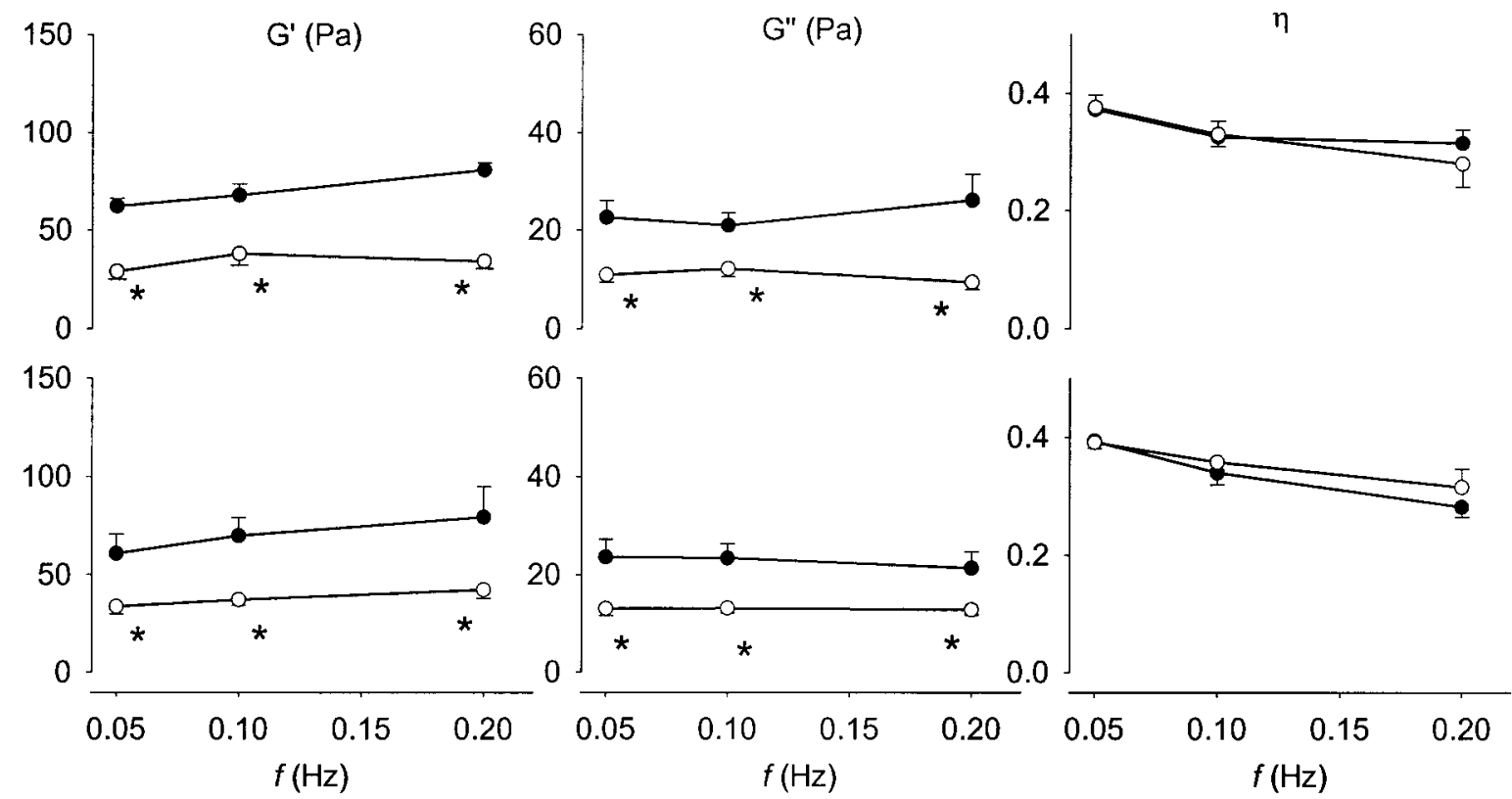

Fig. 9. Control $(\bullet)$ and plateau $(\circ)$ responses from relaxant agonists. Top: dibutyryl cAMP (DBcAMP). Bottom: isoproterenol. In each case, $\mathrm{G}^{\prime}($ left $)$ and $\mathrm{G}^{\prime \prime}$ (middle) are significantly decreased from control values $\left({ }^{*} P<0.05\right.$ ), whereas $\eta$ (right) remains unchanged. Error bars indicate SE; $n=6$ for both cases. 

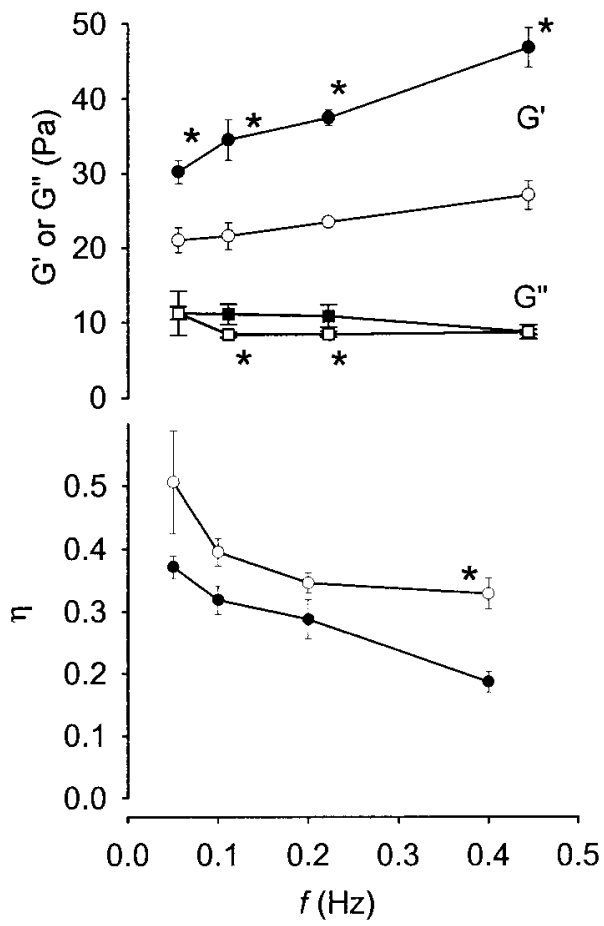

Fig. 10. Changes from baseline (filled symbols) mechanics after administration of $1 \mu \mathrm{g} / \mathrm{ml}$ cytochalasin D (open symbols). G' (circles, top) of the cytoskeleton decreases more than the dissipative behavior, $\mathrm{G}^{\prime \prime}$ (squares, top), leading to a increase in $\eta($ bottom) $(* P<0.05)$. Torque amplitude is $2.9 \mathrm{~Pa}$. Error bars indicate SE; $n=6$.

known as magnetic twisting cytometry, has been widely applied. However, oscillatory magnetic cytometry as reported here represents a technical advance in several respects over its progenitor, the conventional step twisting technique introduced by Wang et al. (55). Using the oscillatory method, we were able to separate the elastic and dissipative behavior over a range of frequencies and to track the time course of mechanical changes of the cytoskeleton. Compared with step twists, oscillatory magnetic twisting was less sensitive to the effects of small numbers of loosely bound beads.

There have been few studies that report the rheological properties of the living cell at different frequencies of mechanical perturbation. These studies have probed the mechanics of either single cells with the use of atomic force microscopy (46) or a large macroscopic quantity of cells suspended in a cone and plate viscometer $(12,23)$. Single cells have also been probed while suspended between glass micropipette attachments to the cell body (47). Schroff et al. (46) used an atomic force microscope to probe the mechanics of rat atrial myocytes. They observed, over a wide range of frequencies, an increasing frequency dependence for the complex modulus amplitude, which is consistent with our findings. Furthermore, they showed that the phase angle between force and displacement was unaltered with stimulation via an increase in intracellular calcium, which is in agreement with the unaltered $\eta$ with contractile stimulation that we report here (since $\eta$ is the tangent of the phase angle; Fig. 8). However, they also reported a slightly increasing phase angle with frequency that is not consistent with our slightly decreasing $\eta$. Measurements of a pellet of cells $\left(>10^{7}\right.$ cells) yields $G^{\prime}$ and $G^{\prime \prime}$ with greater frequency dependence than we show, although $G^{\prime \prime}$ increased only slightly for frequencies below $0.1 \mathrm{~Hz}(12,23)$. Recently, Shue and Brozovich (47) measured oscillatory mechanics (between 5 and $30 \mathrm{~Hz}$ ) of single isolated vascular smooth muscle cells in longitudinal extension and found a positive dependence of stiffness on frequency for the passive muscle, which agrees with the $\mathrm{G}^{\prime}$ behavior we report here in airway smooth muscle cells. However, they could not discern a phase difference when cells were attached to the pipettes vs. when they were not and therefore could not detect frictional losses in the baseline condition. They could detect a phase difference during induced rigor activation, however, and found a decreasing phase angle dependence as we similarly found. In any case, although our results are comparable in many respects with these studies, the methods may probe different structures because of the different methods of coupling to the cell.

In contrast to the few studies of frequency dependence of mechanics in living cells, there have been many studies of the quasi-static elastic properties of cells (Fig. 12). One of the surprising aspects that emerged from studies of cell mechanics is the impressively wide range of moduli reported from the different

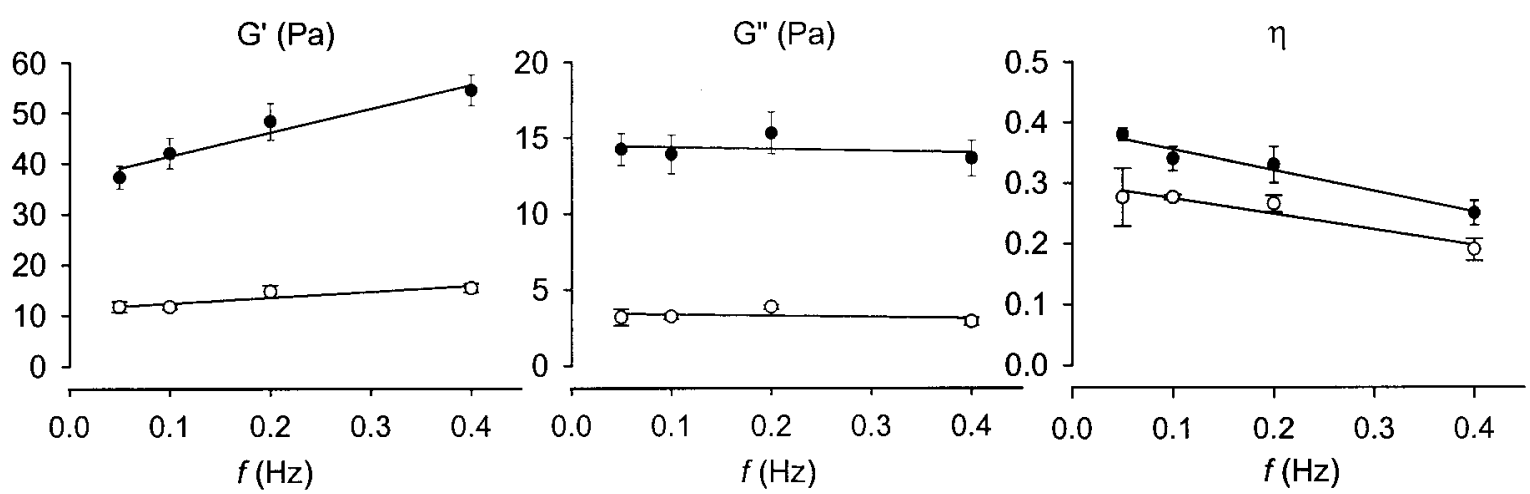

Fig. 11. Comparison of HASM cell mechanics from beads coated with RGD $(\bullet)$ at torque $=2.9$ Pa compared with mechanics from beads coated with acetylated low-density lipoprotein (acLDL; ○) at torque amplitude of $1.9 \mathrm{~Pa} . \mathrm{G}^{\prime}$ (left) and $\mathrm{G}^{\prime \prime}$ (middle) are reduced by a factor of 5 , and $\eta$ (right) is slightly decreased with beads bound via acLDL. 


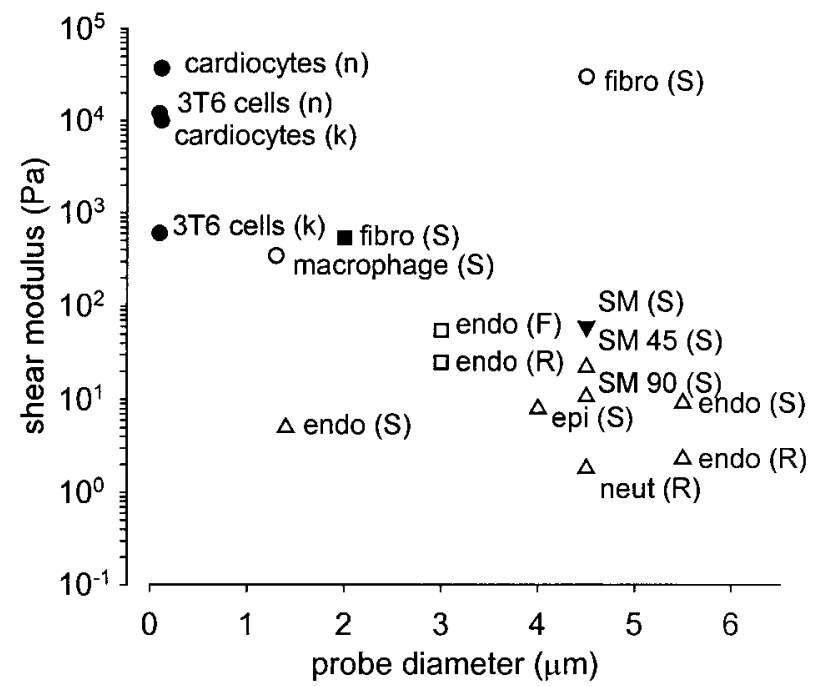

Fig. 12. Comparison of reported elastic properties of different cell types by 6 different techniques under some different conditions adapted from Stamenovic and Coughlin (49). •, Atomic force microscopy of chicken cardiocytes (28) and 3T6 cells (43). ○, Magnetic bead pulling on fibroblasts (fibro) (2) and macrophages (1). n, Cell poking of fibroblasts (38). $\square$, Micropipette aspiration on porcine endothelial cells (endo) (44). $\triangle$, Step magnetic twisting cytometry on endothelial cells $(56,57)$, epithelial cells (epi) (41), neutrophils (neut) (34), and HASM cells (SM) from Hubmayr et al. (29) and from this report at $0^{\circ}$ $(0)$ and $45^{\circ}$ magnetization angle (45). $\mathbf{v}$, Oscillatory magnetometry from this report. Note that values from oscillatory magnetometry are elevated from step magnetometry at both $45^{\circ}$ and $90^{\circ}$, likely due to the sensitivity of the step magnetometry to more weakly bound beads. For oscillatory magnetometry, we make no attempt to derive a shear modulus and include $\mathrm{G}^{\prime}$ as functionally defined by $E q$. 4 . For reports of Young's moduli, we converted to shear moduli with the usual assumption of cell incompressibility, and the shear modulus is one-third that of Young's modulus in this case. S, spread cells; R, rounded cells; $\mathrm{F}$, cells in shear flow; $\mathrm{n}$, cell probed at a thin region; $\mathrm{k}$, cell probed at a thick region.

methods. With few exceptions, the elastic moduli reported in the literature are seen to decrease strongly (exponentially) with the size of the probe (Fig. 12). Although it is expected that the measured mechanical properties would depend on the magnitude and rate of deformation, why moduli should vary so greatly among techniques, and apparently with the probe size, is an intriguing and important question that remains unresolved.

Although there are few studies of the rheology of the living cell at different frequencies, the rheology of networks of cytoskeletal filaments has been a major focus of investigation $(32,36)$. This is not only because of their importance to the living cell but also because models of fiber mechanics based on their thermal motions and interfiber entanglements are theoretically accessible. Indeed, measurements of the oscillatory rheology of reconstituted actin gels over a wide range of frequencies (61) have demonstrated good agreement with theoretical predictions (11). For pure actin gels of high concentration, $\mathrm{G}^{\prime}$ is large in magnitude and is frequency independent, with $\mathrm{G}^{\prime \prime}$ only $1-3 \%$ of $\mathrm{G}^{\prime}$. However, in the presence of proteins such as gelsolin, which acts to shorten actin filaments, $\mathrm{G}^{\prime}$ is greatly reduced and depends on frequency according to a power law with an exponent of nearly 0.15 , close to our value of 0.2 (32). $\mathrm{G}^{\prime \prime}$ is also affected by gelsolin, increasing to a level of about one-quarter that of $\mathrm{G}^{\prime}$, similar to our results in living cells, but also becomes frequency dependent according to a power law, with a slightly greater exponent in contrast to our frequency-independent results (32). There are numerous proteins within the living cell that can alter the mechanical behavior of the cytoskeleton. Indeed, the presence of cross-linking proteins or actin-capping proteins can alter the frequency dependence of $\mathrm{G}^{\prime}$ and $\mathrm{G}^{\prime \prime}$ considerably. Depending on the presence of actin-capping or -binding proteins, the exponent for the frequency dependence can range considerably, from $\sim 0.1$ to $0.75(24,32,35,36)$.

It is also interesting to note that, during constant velocity shearing of reconstituted cytoskeletal gels, energy dissipation is exactly independent of the shear rate (4). Because $\mathrm{G}^{\prime \prime}$ was frequency independent in our results, this means that energy was dissipated independent of the oscillation rate. However, the comparable finding to our results may be fortuitous, since constant velocity shearing undoubtedly leads to filament breaking, which is unlikely in our case. It is nevertheless remarkable that the oscillatory rheology we report here is comparable to oscillatory measurements of networks of cytoskeletal filaments, even though the mechanisms underlying the rheology of the cytoskeleton in the intact cell may be more complex than in purified cytoskeletal filaments. For example, actin gels cannot explain the contractile role of myosin on the mechanical properties.

\section{Contractile Responses}

Using step twists of magnetic beads attached to cultured HASM cells, Hubmayr et al. (29) demonstrated that responses to a wide panel of contracting and relaxing agonists show potencies that rank in the same order as that observed at the level of isolated muscle strips. The contractile response reported here using oscillatory twisting (Fig. 6) also mirrored results reported previously at the level of isolated strips of airway smooth muscle (17-19). We did not observe the transient of $\eta$ that is characteristic of the transition from rapidly cycling cross-bridges to slowly cycling latch bridges (17). However, using a related method of oscillatory bead twisting with better temporal resolution, we have found the $\eta$ transient with the use of magnetic beads in this same cell type; we observed a time constant of $\sim 3 \mathrm{~s}$ for this transient (13), which was too fast for us to resolve by the technique we used in this report. Why the $\eta$ transient is shorter in cultured cells than in isolated muscle strips is not known.

Contractile agonists are known to stimulate actin polymerization and cytoskeletal remodeling in airway smooth muscle cells $(25,27,42)$, but the major part of the response seems to be attributable to the activation of acto-myosin cycling and the formation of acto-myosin cross-links (18). In this connection, we observed no dependence of these responses on the amplitude of the oscillatory bead twisting (data not shown); this sug- 
gests to us that the deformations were not large enough to appreciably perturb acto-myosin cycling (17).

Temporal changes of stiffness $\mathrm{G}^{\prime}$ that were observed in response to challenge with contracting or relaxing agonists are thought to be the shadow of underlying changes in the number of actin-myosin cross-links attached at any moment (19). However, the stiffness measurements reported here do not correspond directly to cross-bridge stiffness, as in high-frequency measurements of isolated muscle strips. There are two reasons. First, the frequency range studied here was chosen to overlap the physiological range associated with the action of breathing, which stretches airway smooth muscle. These frequencies are comparable to or smaller than cross-bridge cycling rates; it is well established that muscle stiffness (measured in longitudinal extension) approximates bridge stiffness only in the limit when the frequency of oscillation is far in excess of the bridge cycling rate (59). Second, in our preparation, the motor protein myosin is connected to the bead through a cytoskeletal matrix that is both deformable and in a continuous state of remodeling. Thus, although the hallmarks of a contractile response are unmistakable, at the level of the bead, the action of myosin may be attenuated. Nonetheless, the contractile response was not small, being able to modulate cell stiffness through a range of fourfold (Fig. 13). Thus, because we probed only the low-frequency range below the cross-bridge cycling rate and because the attachment of the bead to the cytoskeleton via the focal adhesions is well established, it is most reasonable to view the mechanics as that of the cytoskeleton, whose mechanical properties are modulated by the activation state of the contractile machinery.

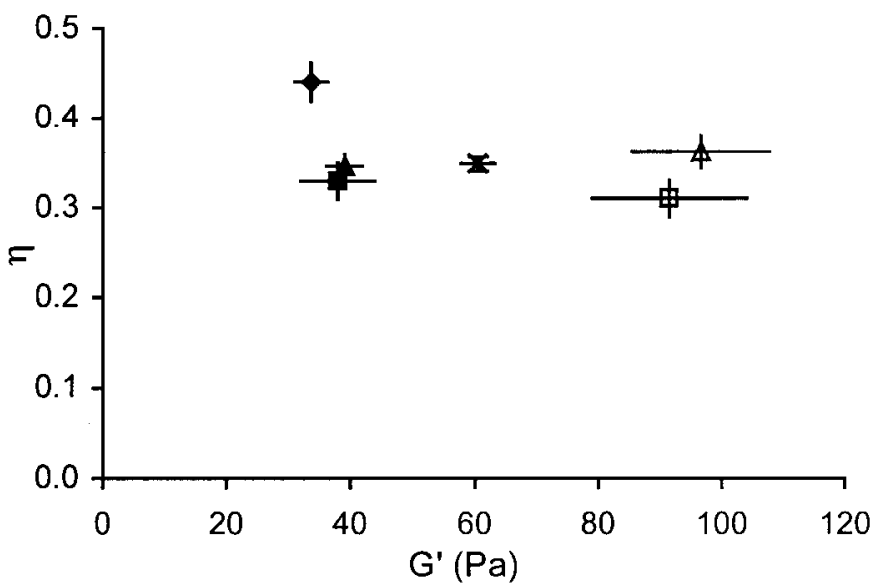

Fig. 13. $\eta$ (Loss tangent) plotted vs. $G^{\prime}$ obtained at torque amplitude of $5.5 \mathrm{~Pa}$ and frequency $=0.1 \mathrm{~Hz}$ in control conditions $(X)$ and after administration of 2 different muscle agonists $\left[10^{-5} \mathrm{M}\right.$ histamine ( $\square$ ) and $\left.10^{-6} \mathrm{M} \mathrm{ET}-1(\triangle)\right]$ and 2 different muscle relaxants $\left[10^{-3} \mathrm{M}\right.$ $\operatorname{DBcAMP}(\mathbf{\square})$ and $10^{-5} \mathrm{M}$ isoproterenol ( $\left.\left.\mathbf{\Delta}\right)\right]$ and after administration of $1 \mu \mathrm{g} / \mathrm{ml}$ cytochalasin $\mathrm{D}(\bullet)$. Each agent was administered to separate cell wells. Data are means (bars indicate SE) from 5-8 wells. Despite large changes in $\mathrm{G}^{\prime}$ during alteration in contractile activation, $\eta$ was unaltered, but disruption of the cytoskeleton by cytochalasin D both decreased $\mathrm{G}^{\prime}$ and increased $\eta$.
Interestingly, except for its short-lived transient, the $\eta$ did not change at all when the contractile state was varied; changes in $\mathrm{G}^{\prime \prime}$ closely tracked those of $\mathrm{G}^{\prime}$ (Figs. 6,8 , and 9). However, $\eta$ was altered with the disruption of actin filaments by cytochalasin D (Figs. 12 and 13). This suggests that constancy of $\eta$ may require an intact cytoskeleton. Together with the constancy of $\mathrm{G}^{\prime \prime}$ with frequency and the constancy of $\eta$ with activation, the frictional stresses during mechanical deformation are suggested to reside within the cytoskeletal fibers or their interactions, rather than in losses attributable to flow of a fluid phase within the cytoplasm.

\section{Structural Damping}

Furthermore, the frequency independence for frictional losses, together with the strong coupling of $\mathrm{G}^{\prime \prime}$ to $\mathrm{G}^{\prime}$, also suggests that the cells that were studied conform well to a phenomenological law that is known as structural damping (7). Structural damping was first recognized by Fung (21) to be important in biological materials and was later elaborated by Fredberg and Stamenovic (20). The near constancy of $\eta$ is consistent with a simple empirical representation of the relation between (rotational) stress and the (rotational) strain

$$
T(\mathrm{f})=\mathrm{G}^{\prime}(1+i \eta) \phi(\mathrm{f})
$$

That is to say, the amplitude of the frictional (out of phase) stress is a fixed fraction, $\eta$, of the elastic stress, $\mathrm{G}^{\prime} \phi$. Because it links frictional loss directly to elastic behavior, structural damping represents a substantial phenomenological simplification. Constancy of $\eta$ implies that frictional energy loss and elastic energy storage are somehow linked to one another at the level of mechanism.

What mechanisms might underlie a frictional stress that is independent of the frequency of deformation and tightly coupled to the elastic stress? Theories such as tensegrity (31) or percolation (16) attribute cell elasticity to the interconnected nature of the cytoskeleton but have not yet been connected with energy dissipation. Common models employing rate-dependent Newtonian viscosity, including one or a few viscosity elements $(2,26,45,51)$, are insufficient to describe rate-independent energy dissipation. Because of the similarity of our findings to the behavior for reconstituted actin gels, candidate mechanisms could be those describing fiber networks, such as fiber reptation as introduced by de Gennes (10) and modified later by others (15). These theories derive elastic and dissipative behaviors from the entangled thermal motions of the cytoskeletal filaments and have successfully described many of the rheological features of reconstituted actin gels (33). However, these models do not incorporate stable associations that are formed between fibers via cross-linking molecules that are abundant in living cells (e.g., filamin, $\alpha$-actinin), and these models also do not account for the important effects of contractile proteins such as myosin that we demonstrate here.

However, there are other mechanisms that may be present within the cytoskeleton that are inherently 
rate independent. For example, Coulomb friction associated with fiber sliding on fiber would be rate independent, as would be the discontinuous breaking and reforming of weak bonds that occurs during protein folding and unfolding $(48,52)$. In any case, we cannot discriminate among these possible mechanisms. Thus the molecular mechanism responsible for frequencyindependent energy dissipation and for coupling of that dissipation to elastic energy storage remains an unanswered question.

In summary, we have shown here that, in the living airway smooth muscle cell, deformation of the cytoskeleton is dominated by elastic stresses, but frictional stresses are substantial, about one-third of elastic stresses. Brisk contractile and relaxing responses were readily apparent in this culture system, altering both elastic and frictional stresses. These frictional stresses do not likely arise from the flow of a fluid phase within the cytoplasm but, rather, may arise from dissipative mechanisms from within the cytoskeleton. Furthermore, the frictional stresses were frequency invariant and were tightly coupled to the elastic stresses, but the mechanism of this coupling is not known.

We gratefully acknowledge the support from Drs. W. Moeller and colleagues at the Forschungszentrum für Umwelt und Gesundheit National Research Center for Environment and Health Institute for Inhalation Biology, D/85764 Oberschleisseim, Germany, for providing the ferrimagnetic beads. We thank Ning Wang for helpful discussions, Stephanie Shore for support, and Paul Moore, Joseph Abraham, Igor Schwartzmann, and In Lim for experimental assistance. We also gratefully acknowledge Rey Panettieri of the University of Pennsylvania for providing the cells.

This work was supported by National Institutes of Health. G. Maksym was supported by a National Sciences and Engineering Research Council Canada fellowship. B. Fabry was supported by a Deutsche Forschungsgemeinschaft fellowship.

\section{REFERENCES}

1. Bausch AR, Moller W, and Sackmann E. Measurement of local viscoelasticity and forces in living cells by magnetic tweezers. Biophys J 76: 573-579, 1999.

2. Bausch AR, Ziemann F, Boulbitch AA, Jacobson K, and Sackmann E. Local measurements of viscoelastic parameters of adherent cell surfaces by magnetic bead microrheometry. Biophys J 75: 2038-2049, 1998.

3. Bereiter-Hahn J, Karl I, Luers H, and Voth M. Mechanical basis of cell shape: investigations with the scanning acoustic microscope. Biochem Cell Biol 73: 337-348, 1995.

4. Buxbaum RE, Dennerll T, Weiss S, and Heidemann SR. $\mathrm{F}$-actin and microtubule suspensions as indeterminate fluids. Science 235: 1511-1514, 1987.

5. Chen CS, Mrksich M, Huang S, Whitesides GM, and Ingber DE. Geometric control of cell life and death. Science 276: 14251428, 1997.

6. Chicurel ME, Singer RH, Meyer CJ, and Ingber DE. Integrin binding and mechanical tension induce movement of mRNA and ribosomes to focal adhesions. Nature 392: 730-733, 1998.

7. Crandall SH. The role of damping in vibration theory. J Sound Vib 11: 3-18, 1970.

8. Crick FHC. The physical properties of cytoplasm. A study by means of the magnetic particle method. Part 2. Theoretical treatment. Exp Cell Res 1: 505-533, 1950.

9. Crick FHC and Hughes AFW. The physical properties of cytoplasm. A study by means of the magnetic particle method. Part 1. Exp Cell Res 1: 37-80, 1950.

10. De Gennes PG. Reptation of polymer chain in the presence of fixed obstacles. J Chem Phys 55: 572-579, 1971.
11. Doi M and Edwards SF. The Theory of Polymer Dynamics. New York: Oxford Univ. Press, 1986.

12. Eichinger L, Koppel B, Noegel AA, Schleicher M, Schliwa M, Weijer K, Witke W, and Janmey PA. Mechanical perturbation elicits a phenotypic difference between Dictyostelium wild-type cells and cytoskeletal mutants. Biophys $J$ 70: 10541060, 1996.

13. Fabry B, Maksym GN, Butler JP, and Fredberg JJ. Contractile response of human airway smooth muscle cells in culture (Abstract). Am J Respir Crit Care Med 161: A472, 2000.

14. Fabry B, Maksym GN, Hubmayr RD, Butler JP, and Fredberg JJ. Implications of heterogeneous bead behavior on cell mechanical properties measured with magnetic twisting cytometry. J Magnetism Magnetic Materials 194: 120-125, 1999.

15. Ferry JD. Viscoelastic Properties of Polymers. New York: Wiley, 1980.

16. Forgacs G. On the possible role of cytoskeletal filamentous networks in intracellular signaling: an approach based on percolation. J Cell Sci 108: 2131-2143, 1995.

17. Fredberg JJ, Inouye D, Miller B, Nathan M, Jafari S, Raboudi SH, Butler JP, and Shore SA. Airway smooth muscle, tidal stretches, and dynamically determined contractile states. Am J Respir Crit Care Med 156: 1752-1759, 1997.

18. Fredberg JJ, Inouye DS, Mijailovich SM, and Butler JP. Perturbed equilibrium of myosin binding in airway smooth muscle and its implications in bronchospasm. Am J Respir Crit Care Med 159: 959-967, 1999

19. Fredberg JJ, Jones KA, Nathan M, Raboudi S, Prakash YS, Shore SA, Butler JP, and Sieck GC. Friction in airway smooth muscle: mechanism, latch, and implications in asthma. J Appl Physiol 81: 2703-2712, 1996.

20. Fredberg JJ and Stamenovic D. On the imperfect elasticity of lung tissue. J Appl Physiol 67: 2408-2419, 1989.

21. Fung YC. Biomechanics, Mechanical Properties of Living Tissues. New York: Springer-Verlag, 1984.

22. Galbraith CG, Skalak R, and Chien S. Shear stress induces spatial reorganization of the endothelial cell cytoskeleton. Cell Motil Cytoskeleton 40: 317-330, 1998.

23. Goldmann WH and Ezzell RM. Viscoelasticity in wild-type and vinculin-deficient (5.51) mouse F9 embryonic carcinoma cells examined by atomic force microscopy and rheology. Exp Cell Res 226: 234-237, 1996.

24. Goldmann WH, Tempel M, Sprenger I, Isenberg G, and Ezzell RM. Viscoelasticity of actin-gelsolin networks in the presence of filamin. Eur J Biochem 246: 373-379, 1997.

25. Gunst SJ, Meiss RA, Wu MF, and Rowe M. Mechanisms for the mechanical plasticity of tracheal smooth muscle. Am J Physiol Cell Physiol 268: C1267-C1276, 1995.

26. Heidemann SR, Kaech S, Buxbaum RE, and Matus A. Direct observations of the mechanical behaviors of the cytoskeleton in living fibroblasts. J Cell Biol 145: 109-122, 1999.

27. Hirshman CA and Emala CW. Actin reorganization in airway smooth muscle cells involves Gq and Gi-2 activation of Rho. Am J Physiol Lung Cell Mol Physiol 277: L653-L661, 1999.

28. Hofmann UG, Rotsch C, Parak WJ, and Radmacher M. Investigating the cytoskeleton of chicken cardiocytes with the atomic force microscope. J Struct Biol 119: 84-91, 1997.

29. Hubmayr RD, Shore SA, Fredberg JJ, Planus E, Panettieri RA Jr, Moller W, Heyder J, and Wang N. Pharmacological activation changes stiffness of cultured human airway smooth muscle cells. Am J Physiol Cell Physiol 271: C1660C1668, 1996.

30. Ingber D. Integrins as mechanochemical transducers. Curr Opin Cell Biol 3: 841-848, 1991.

31. Ingber DE. Tensegrity: the architectural basis of cellular mechanotransduction. Annu Rev Physiol 59: 575-599, 1997.

32. Janmey PA, Hvidt S, Kas J, Lerche D, Maggs A, Sackmann E, Schliwa M, and Stossel TP. The mechanical properties of actin gels. Elastic modulus and filament motions. $J$ Biol Chem 269: 32503-32513, 1994

33. Kas J, Strey H, Tang JX, Finger D, Ezzell R, Sackmann E, and Janmey PA. F-actin, a model polymer for semiflexible chains in dilute, semidilute, and liquid crystalline solutions. Biophys J 70: 609-625, 1996. 
34. Kimura Y, Wang N, Butler JP, Fredberg JJ, and Doerschuk CM. Mechanical properties of quiescent and activated neutrophils measured through CD11a compared to CD11b (Abstract). Am J Respir Crit Care Med 155: A294, 1997.

35. Leterrier JF, Kas J, Hartwig J, Vegners R, and Janmey PA. Mechanical effects of neurofilament cross-bridges. Modulation by phosphorylation, lipids, and interactions with F-actin. J Biol Chem 271: 15687-15694, 1996.

36. Palmer A, Xu J, Kuo SC, and Wirtz D. Diffusing wave spectroscopy microrheology of actin filament networks. Biophys $J$ 76: 1063-1071, 1999.

37. Panettieri RA, Murray RK, DePalo LR, Yadvish PA, and Kotlikoff MI. A human airway smooth muscle cell line that retains physiological responsiveness. Am J Physiol Cell Physiol 256: C329-C335, 1989.

38. Petersen NO, McConnaughey WB, and Elson EL. Dependence of locally measured cellular deformability on position on the cell, temperature, and cytochalasin B. Proc Natl Acad Sci USA 79: 5327-5331, 1982.

39. Plopper GE, McNamee HP, Dike LE, Bojanowski K, and Ingber DE. Convergence of integrin and growth factor receptor signaling pathways within the focal adhesion complex. Mol Biol Cell 6: 1349-1365, 1995.

40. Pommerenke H, Schreiber E, Durr F, Nebe B, Hahnel C, Moller W, and Rychly J. Stimulation of integrin receptors using a magnetic drag force device induces an intracellular free calcium response. Eur J Cell Biol 70: 157-164, 1996.

41. Potard US, Butler JP, and Wang N. Cytoskeletal mechanics in confluent epithelial cells probed through integrins and E-cadherins. Am J Physiol Cell Physiol 272: C1654-C1663, 1997.

42. Pratusevich VR, Seow CY, and Ford LE. Plasticity in canine airway smooth muscle. J Gen Physiol 105: 73-94, 1995.

43. Ricci D, Tedesco M, and Grattarola M. Mechanical and morphological properties of living 3T6 cells probed via scanning force microscopy. Microsc Res Tech 36: 165-171, 1997.

44. Sato M, Ohshima N, and Nerem RM. Viscoelastic properties of cultured porcine aortic endothelial cells exposed to shear stress. J Biomech 29: 461-467, 1996.

45. Schmid-Schonbein GW, Sung KL, Tozeren H, Skalak R, and Chien S. Passive mechanical properties of human leukocytes. Biophys J 36: 243-256, 1981.

46. Shroff SG, Saner DR, and Lal R. Dynamic micromechanical properties of cultured rat atrial myocytes measured by atomic force microscopy. Am J Physiol Cell Physiol 269: C286-C292, 1995
47. Shue G and Brozovich FV. The frequency response of smooth muscle stiffness during $\mathrm{Ca}^{2+}$-activated contraction. Biophys $J$ 76: 2361-2369, 1999.

48. Smith BL, Schaffer TE, Viani M, Thompson JB, Frederick NA, Kindt J, Belcher A, Stucky GD, Morse DE, and Hansma PK. Molecular mechanistic origin of the toughness of natural adhesives, fibres and composites. Nature 399: 761-763, 1999.

49. Stamenovic D and Coughlin MF. The role of prestress and architecture of the cytoskeleton and deformability of cytoskeletal filaments in mechanics of adherent cells: a quantitative analysis. J Theor Biol 201: 63-74, 1999.

50. Stossel TP. On the crawling of animal cells. Science 260: 10861094, 1993

51. Thoumine O, Cardoso O, and Meister JJ. Changes in the mechanical properties of fibroblasts during spreading: a micromanipulation study. Eur Biophys J 28: 222-234, 1999.

52. Tskhovrebova L, Trinick J, Sleep JA, and Simmons RM. Elasticity and unfolding of single molecules of the giant muscle protein titin. Nature 387: 308-312, 1997.

53. Valberg PA. Magnetometry of ingested particles in pulmonary macrophages. Science 224: 513-516, 1984.

54. Valberg PA and Butler JP. Magnetic particle motions within living cells. Physical theory and techniques. Biophys $J$ 52: 537550,1987

55. Wang N, Butler JP, and Ingber DE. Mechanotransduction across the cell surface and through the cytoskeleton. Science 260: 1124-1127, 1993.

56. Wang $\mathbf{N}$ and Ingber DE. Control of cytoskeletal mechanics by extracellular matrix, cell shape, and mechanical tension. Biophys J 66: 2181-2189, 1994

57. Wang $\mathbf{N}$ and Ingber DE. Probing transmembrane mechanical coupling and cytomechanics using magnetic twisting cytometry. Biochem Cell Biol 73: 327-335, 1995.

58. Wang YL and Pelham RJ Jr. Preparation of a flexible, porous polyacrylamide substrate for mechanical studies of cultured cells. Methods Enzymol 298: 489-496, 1998.

59. Warshaw DM, Rees DD, and Fay FS. Characterization of cross-bridge elasticity and kinetics of cross-bridge cycling during force development in single smooth muscle cells. J Gen Physiol 91: 761-779, 1988.

60. Yanai M, Butler JP, Suzuki T, Kanda A, Kurachi M, Tashiro H, and Sasaki H. Intracellular elasticity and viscosity in the body, leading, and trailing regions of locomoting neutrophils. Am J Physiol Cell Physiol 277: C432-C440, 1999.

61. Ziemann F, Radler J, and Sackmann E. Local measurements of viscoelastic moduli of entangled actin networks using an oscillating magnetic bead micro-rheometer. Biophys J 66: 22102216,1994 